\title{
VERY EARLY ULTRAVIOLET AND OPTICAL OBSERVATIONS OF THE TYPE Ia SUPERNOVA 2009ig
}

\author{
Ryan J. Foley ${ }^{1,16}$, P. J. Challis ${ }^{1}$, A. V. Filippenko ${ }^{2}$, M. Ganeshalingam ${ }^{2}$, W. Landsman ${ }^{3}$, W. Li ${ }^{2}$, G. H. Marion ${ }^{1}$,

 \\ J. S. Kalirai ${ }^{10}$, R. P. Kirshner ${ }^{1}$, A. Stockton ${ }^{11}$, E. J. Tollerud ${ }^{12}$, J. Vinkó ${ }^{13,14}$, J. C. Wheeler ${ }^{14}$, And J.-H. Woo $^{15}$ \\ ${ }^{1}$ Harvard-Smithsonian Center for Astrophysics, 60 Garden Street, Cambridge, MA 02138, USA; rfoley @ cfa.harvard.edu. \\ ${ }^{2}$ Department of Astronomy, University of California, Berkeley, CA 94720-3411, USA \\ ${ }^{3}$ NASA Goddard Space Flight Center, Greenbelt, MD 20771, USA \\ ${ }^{4}$ Department of Astronomy, University of Virginia, Charlottesville, VA 22904-4325, USA \\ ${ }^{5}$ Department of Physics, University of California, Santa Barbara, CA 93106-9530, USA \\ ${ }^{6}$ Physics Division, Lawrence Berkeley National Laboratory, 1 Cyclotron Road, Berkeley, CA 94720, USA \\ ${ }^{7}$ Department of Physics, University of California, 366 LeConte Hall, Berkeley, CA 94720-7300, USA \\ ${ }^{8}$ UCO/Lick Observatory, University of California, Santa Cruz, CA 95064, USA \\ ${ }^{9}$ Steward Observatory, University of Arizona, 933 North Cherry Avenue, Tucson, AZ 85721, USA \\ ${ }^{10}$ Space Telescope Science Institute, 3700 San Martin Drive, Baltimore, MD 21218, USA \\ ${ }^{11}$ Institute for Astronomy, University of Hawaii, Honolulu, HI 96822, USA \\ 12 Center for Cosmology, Department of Physics and Astronomy, 4129 Frederick Reines Hall, University of California, Irvine, CA 92697, USA \\ ${ }^{13}$ Department of Optics \& Quantum Electronics, University of Szeged, Dóm tèr 9, Szeged H-6720, Hungary \\ ${ }^{14}$ Astronomy Department, University of Texas, Austin, TX 78712, USA \\ ${ }^{15}$ Astronomy Program, Department of Physics and Astronomy, Seoul National University, Seoul, 151-742, Korea \\ Received 2011 September 4; accepted 2011 October 25; published 2011 December 13
}

\begin{abstract}
Supernova (SN) 2009ig was discovered $17 \mathrm{hr}$ after explosion by the Lick Observatory Supernova Search, promptly classified as a normal Type Ia SN (SN Ia), peaked at $V=13.5 \mathrm{mag}$, and was equatorial, making it one of the foremost SNe for intensive study in the last decade. Here, we present ultraviolet (UV) and optical observations of SN 2009ig, starting about 1 day after explosion until around maximum brightness. Our data include excellent UV and optical light curves, 25 premaximum optical spectra, and $8 \mathrm{UV}$ spectra, including the earliest UV spectrum ever obtained of an SN Ia. SN 2009ig is a relatively normal SN Ia, but does display high-velocity ejecta-the ejecta velocity measured in our earliest spectra $\left(v \approx-23,000 \mathrm{~km} \mathrm{~s}^{-1}\right.$ for Si II $\left.\lambda 6355\right)$ is the highest yet measured in an $\mathrm{SN}$ Ia. The spectral evolution is very dramatic at times earlier than 12 days before maximum brightness, but slows after that time. The early-time data provide a precise measurement of $17.13 \pm 0.07$ days for the SN rise time. The optical color curves and early-time spectra are significantly different from template light curves and spectra used for light-curve fitting and $K$-corrections, indicating that the template light curves and spectra do not properly represent all SNe Ia at very early times. In the age of wide-angle sky surveys, SNe like SN 2009ig that are nearby, bright, well positioned, and promptly discovered will still be rare. As shown with SN 2009ig, detailed studies of single events can provide significantly more information for testing systematic uncertainties related to SN Ia distance estimates and constraining progenitor and explosion models than large samples of more distant SNe.
\end{abstract}

Key words: supernovae: general - supernovae: individual (SN 2009g)

Online-only material: color figures

\section{INTRODUCTION}

Type Ia supernovae ( $\mathrm{SNe}$ Ia) are exceptionally good distance indicators, allowing precise measurements of various cosmological parameters, including the first significant constraints on $\Omega_{\Lambda}$ (e.g., Riess et al. 1998, 2007; Perlmutter et al. 1999; Wood-Vasey et al. 2007; Hicken et al. 2009; Kessler et al. 2009; Amanullah et al. 2010; Conley et al. 2011; Suzuki et al. 2011). The progenitor system and explosion mechanism are generally known (a thermonuclear explosion of a $\mathrm{C} / \mathrm{O}$ white dwarf (WD) in a binary system; Hoyle \& Fowler 1960; Colgate \& McKee 1969; Nomoto et al. 1984; Woosley et al. 1986), but the specifics of the various models-such as whether the progenitor comes from a single- or double-degenerate system-are ill constrained (e.g., Hillebrandt \& Niemeyer 2000; Howell 2011). Many studies of individual peculiar SNe Ia, which can probe the extremities of the models or show what a normal SN Ia is not, have been conducted over the years (e.g., Li et al. 2001,

\footnotetext{
${ }^{16}$ Clay Fellow.
}

2003; Hamuy et al. 2003; Howell et al. 2006; Foley et al. 2009, 2010), but intense studies of individual normal SNe Ia are relatively rare. Systematic studies of large samples of normal SNe Ia can provide important constraints for the models, but extremely detailed observations of even a single normal object can be as constraining as hundreds of objects with much lower quality data.

The ultraviolet (UV) portion of an SN Ia spectral energy distribution (SED) provides a bounty of information about the explosions of SNe Ia. UV spectra are the most constraining data for determining the effects of temperature, density, and nonthermal ionization (e.g., Höflich et al. 1998; Lentz et al. 2001). SN Ia UV spectra are dominated by a forest of overlapping lines from Fe-group elements. UV photons are repeatedly absorbed and re-emitted in those lines and gradually scattered redward where lower opacities allow them to escape. Therefore, the UV is crucial to the formation of the optical SED of SNe Ia (Sauer et al. 2008) and extremely sensitive to both the progenitor composition and explosion mechanism. Observations of the UV directly probe the composition of the outermost layers of ejecta 
which are transparent at optical wavelengths soon after explosion. Furthermore, Kasen (2010) showed that single-degenerate progenitor systems should have strong UV emission at early times from the SN ejecta interacting with the companion star; therefore, early UV observations directly test progenitor models.

At optical wavelengths SNe Ia are remarkably uniform in luminosity ( $\sigma \approx 0.16 \mathrm{mag}$; Mandel et al. 2011), after correcting for light-curve shape and color. This relationship extends to the $U$ band and UV, but with larger scatter (Jha et al. 2006; Brown et al. 2010). The scatter in the optical can be further reduced if one accounts for the correlation between intrinsic color and ejecta velocity (Foley \& Kasen 2011). Along with the observed $B-V$ color of SNe Ia (Pignata et al. 2008; Wang et al. 2009a), their intrinsic $B-V$ color correlates strongly with velocity, with intrinsically redder objects having high velocity (Foley \& Kasen 2011; Foley et al. 2011). This is explained as additional line blanketing in the $B$ band of the high-velocity objects, and this trend should extend to the UV (Foley \& Kasen 2011). Ganeshalingam et al. (2011) found that higher-velocity $\mathrm{SNe}$ Ia tend to have faster $B$-band rise times than lower-velocity $\mathrm{SNe}$ Ia $(\Delta t=1.4$ days), but this is not the case for $V$-band rise times.

SNe Ia bright enough to be observed by the International Ultraviolet Explorer (IUE) were rare (Foley et al. 2008), and only one object has a published high signal-to-noise ratio (S/N) Hubble Space Telescope (HST) spectrum near maximum light covering wavelengths $\lesssim 2900 \AA$ A: SN 1992A (Kirshner et al. 1993), observed nearly two decades ago. Swift has obtained spectra of several SNe Ia, but because of typically short exposure times, they are generally of poor quality (Bufano et al. 2009). In Cycle 17, HST obtained a single spectrum of $30 \mathrm{SNe}$ Ia (GO-11721; PI Ellis); however, because of the choice of grating, these spectra do not probe wavelengths shorter than $\sim 2900 \AA$ (Cooke et al. 2011). In total, there have been seven SNe Ia with published premaximum spectra probing wavelengths shortward of $\sim 2900 \AA$ (SNe $1980 \mathrm{~N}, 1986 \mathrm{G}, 1989 \mathrm{~B}, 1990 \mathrm{~N}, 1991 \mathrm{~T}$, 1992A, and 2005; cf. Jeffery et al. 1992; Kirshner et al. 1993; Foley et al. 2008; Bufano et al. 2009).

SN 2009ig was discovered at an unfiltered magnitude of 17.5, 4 mag below peak, on 2009 August 20.48 (UT dates are used throughout this paper) by Kleiser et al. (2009) during the Lick Observatory Supernova Search with the 0.76 m Katzman Automatic Imaging Telescope (KAIT; Filippenko et al. 2001). There was no object detected on 2009 August 16.46 to a limit of 18.7 mag. It was discovered in NGC 1015, an SBa galaxy at $c z=$ $2629 \mathrm{~km} \mathrm{~s}^{-1}$ (Wong et al. 2006) with distance $D=33.1 \mathrm{Mpc}$ $(\mu=32.6 \pm 0.4 \mathrm{mag})$ from a Tully-Fisher measurement (Tully 1988).

Navasardyan et al. (2009) obtained an optical spectrum of SN 2009ig on 2009 August 21.08, only 0.7 days after discovery, and determined that it was a young SN Ia. Clearly, SN 2009ig was discovered very shortly after explosion. We triggered multiple programs to study the photometric and spectroscopic evolution of the object, its circumstellar environment, its spectropolarimetry, its energetics, and other aspects. Here we focus on the early-time UV and optical SN spectroscopy and photometry.

We present our UV and optical data in Section 2, and derive basic maximum-light parameters for SN 2009ig in Section 3. The UV spectra are discussed in Section 4. In Sections 5 and 6, we compare the early-time photometry and spectroscopy, respectively, to that of other objects and to models. We discuss our findings and summarize our conclusions in Section 7.

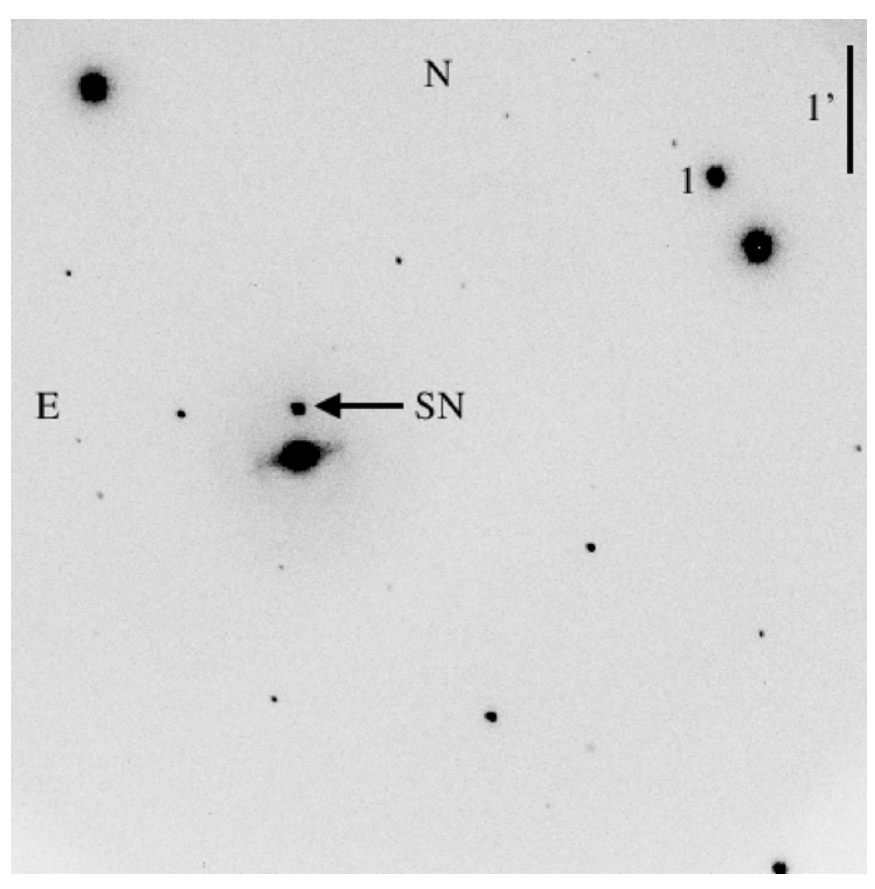

Figure 1. KAIT $R$-band image of SN 2009ig and its host galaxy, NGC 1015. The field of view (FOV) is 6.7 $\times 6$ 6.7. The $\mathrm{SN}$ and comparison star are marked.

\section{OBSERVATIONS AND DATA REDUCTION}

\subsection{Optical and Ultraviolet Photometry}

Broadband BVRI photometry of SN 2009ig was obtained using KAIT with the KAIT4 filters set (Ganeshalingam et al. 2010) at Lick Observatory starting on 2009 August 21.5 (about two weeks before maximum light). Within a day of the discovery of SN 2009ig, KAIT was programmed to observe the field with a nightly cadence to sample the rise and maximum of the light curves. Two weeks after maximum light the cadence was changed to every 3-4 days. In total, we have 55 photometry epochs. A finder chart of SN 2009ig, its host galaxy, and a comparison star is shown in Figure 1.

The data were reduced using a mostly automated pipeline developed for KAIT images (Ganeshalingam et al. 2010). Images were bias-corrected and flat-fielded at the telescope. Using galaxy templates obtained a year and a half after discovery, the data images were galaxy subtracted to remove galaxy flux at the position of the SN. The flux of the SN and the local field star were measured using the point-spread function fitting photometry package DAOPHOT in IRAF. ${ }^{17}$ Instrumental magnitudes were color-corrected to the Landolt (1992) system using the average color terms measured on multiple photometric nights as presented by Ganeshalingam et al. (2010). The magnitudes of the local field standard were calibrated against Landolt (1992) standards on seven photometric nights.

The field of SN 2009ig suffers from a dearth of local standards that are bright enough to measure reliably, but not so bright that they saturate the detector. After trying combinations of available standards, we find that the smoothest, most believable light curves are obtained using a single, bright comparison star whose photometry is given in Table 1 . We caution that this leaves our final light curves susceptible to a systematic offset

\footnotetext{
17 IRAF: The Image Reduction and Analysis Facility is distributed by the National Optical Astronomy Observatory, which is operated by the

Association of Universities for Research in Astronomy, Inc., under cooperative
} agreement with the National Science Foundation (NSF). 
Table 1

Photometry of SN 2009ig Standard Star

\begin{tabular}{|c|c|c|c|c|c|c|c|c|c|c|}
\hline ID & $\alpha(\mathrm{J} 2000)$ & $\delta(\mathrm{J} 2000)$ & $B\left(\sigma_{B}\right)$ & $N_{B}$ & $V\left(\sigma_{V}\right)$ & $N_{V}$ & $R\left(\sigma_{R}\right)$ & $N_{R}$ & $I\left(\sigma_{I}\right)$ & $\overline{N_{I}}$ \\
\hline 1 & $02: 37: 58.65$ & $-01: 16: 56.4$ & $13.637(004)$ & 6 & $13.039(003)$ & 6 & $12.829(007)$ & 4 & $12.426(015)$ & 3 \\
\hline
\end{tabular}

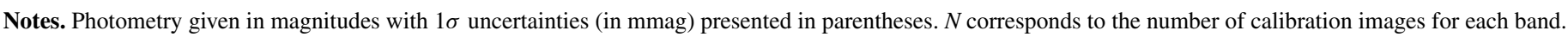

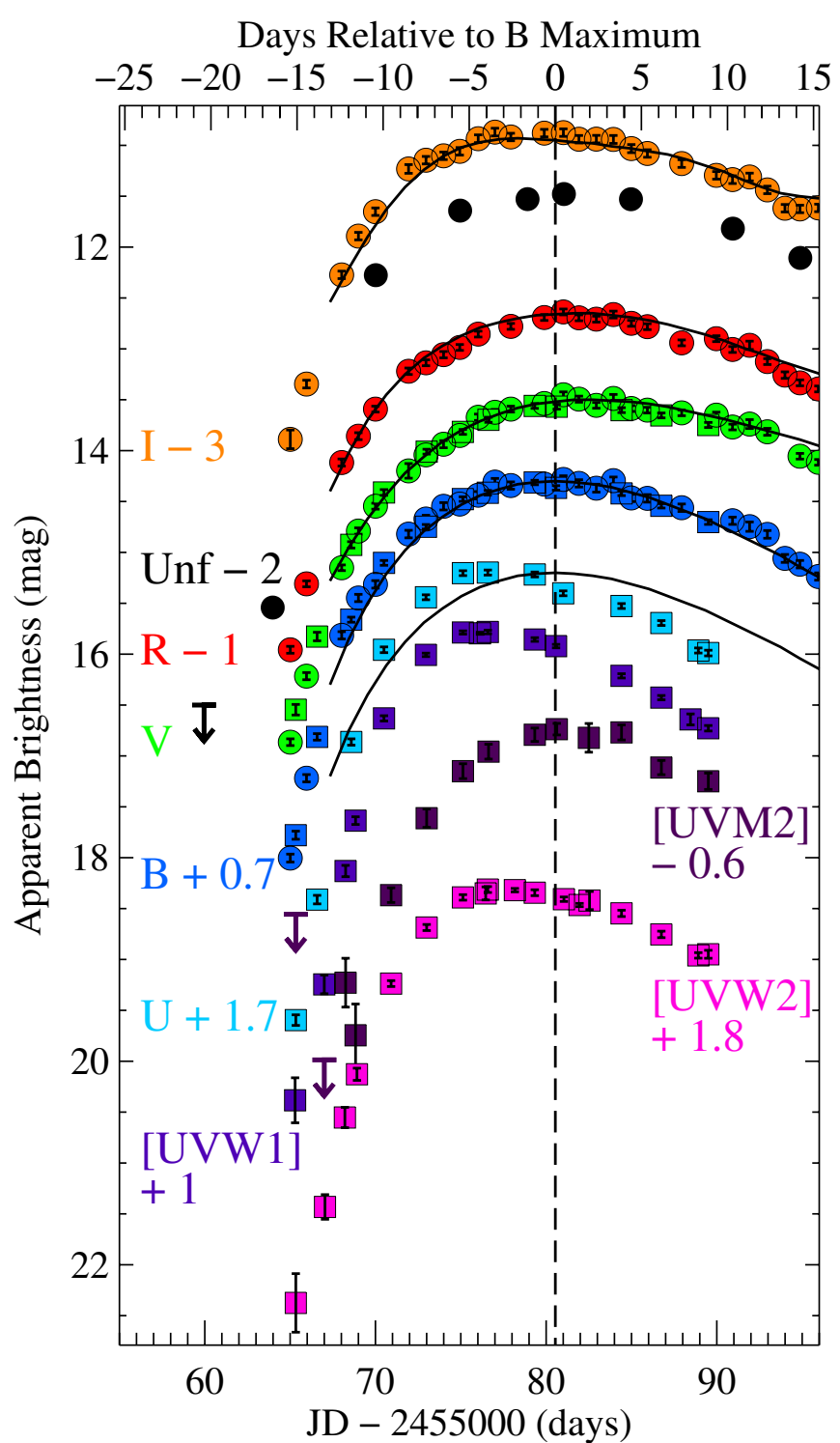

Figure 2. [UVW2][UVM2][UVW1] $U B V R I$ (fuchsia, dark purple, navy, cyan, blue, green, red, and orange, respectively; circles and squares correspond to KAIT and Swift/UVOT data, respectively) and unfiltered (black circles, with the label "Unf") light curves of SN 2009ig. Our unfiltered magnitudes closely approximate the $R$ band. The uncertainties for most data points are smaller than the plotted symbols. Also shown are comparison $U B V R I$ template light curves of SN 2005cf (Wang et al. 2009b), stretched by a factor of 1.1 .

(A color version of this figure is available in the online journal.)

from an incorrect determination of the Landolt magnitude of the comparison star, but should not change the overall shape of our light curves. KAIT photometry of SN 2009ig is presented in Figure 2 and Table 2.

The Swift team initiated target-of-opportunity observations of SN 2009ig with the Ultraviolet/Optical Telescope (UVOT; Roming et al. 2005) and the X-ray Telescope (Burrows et al. 2005) on board the Swift gamma-ray burst satellite (Gehrels et al. 2004) beginning 2009 August 21.8. We performed digital image subtraction on all of the UVOT data using the final epoch as a template to remove host-galaxy contamination with the ISIS software package (Alard 2000). The $U_{-}, B$-, and $V$-band data were then reduced using the calibration technique described by $\mathrm{Li}$ et al. (2006), while [UVW2], [UVM2], and [UVW1] (corresponding to central/effective wavelengths of 1941/3064, 2248/2360, and 2605/3050 A, respectively; Brown et al. 2010) photometry was obtained using the zero points from Poole et al. (2008).

The three bluest UVOT filters all have a significant wing that extends to optical wavelengths. This "red leak" is particularly problematic for SNe Ia, which have SEDs that peak in the optical and significantly depressed UV flux relative to the optical flux. As a result, a significant fraction of the flux measured in the UV filters comes from the optical portion of the SED. Brown et al. (2010) provide red-leak corrections for the UVOT filters. However, these corrections were calculated from the $t=+5$ day SN 1992A spectrum, which is the single published high $\mathrm{S} / \mathrm{N}$ UV near-maximum SN Ia spectrum (Kirshner et al. 1993). SN Ia SEDs evolve with time, and especially quickly for the phases we examine here. We therefore do not want to make a single correction to our data. Unfortunately, there are no other suitable, published SN Ia spectra from which we could make red-leak corrections. As a result, we present the UVOT data without a red-leak correction.

The results of our UVOT analysis are displayed in Figure 2 and presented in Table 3.

To correct the KAIT and Swift photometry for small systematic differences due to different instrumental response functions, we apply $S$-corrections to put our optical photometry on the standard system following the method of Stritzinger et al. (2002). This procedure is done in addition to standard color-corrections which are appropriate for stellar SEDs, but can be inaccurate for the SED of an SN. The instrumental response function includes the effects of the filter transmission, quantum efficiency of the detector, mirror reflectivity, and the atmospheric transmission in the case of ground-based telescopes. The instrumental response functions for our KAIT photometry correspond to KAIT4 in Ganeshalingam et al. (2010). We calculate synthetic magnitudes for the SN using the Bessel transmission curves of Stritzinger et al. (2005) and the instrumental response functions using our optical spectral series for SN 2009ig. In instances where our spectra of SN 2009ig do not cover the $B$ band (e.g., around maximum light), we instead use spectra from the Hsiao et al. (2007) spectral series at the appropriate phase warped using a third-order spline to match the colors of the KAIT natural system photometry. The difference between the Bessel synthetic magnitude and the color-corrected instrumental synthetic magnitude is the $S$-correction (see Stritzinger et al. 2002; Wang et al. 2009 b for more details on computing $S$-corrections). We fit splines to the KAIT $B$ and $V$ data to estimate the KAIT magnitudes at the time of the Swift observations in these bands. The residuals of KAIT minus Swift photometry in the $B(V)$ band have a mean and standard deviation of $-0.01 \mathrm{mag}(-0.03 \mathrm{mag})$ and $0.07 \mathrm{mag}$ (0.06 mag), respectively. 
Table 2

KAIT Photometry and $S$-Corrections for SN 2009ig

\begin{tabular}{|c|c|c|c|c|c|c|c|c|}
\hline JD & $\begin{array}{c}B \\
(\mathrm{mag})\end{array}$ & $\begin{array}{c}S_{B} \\
(\mathrm{mag})\end{array}$ & $\begin{array}{c}V \\
(\mathrm{mag})\end{array}$ & $\begin{array}{c}S_{V} \\
(\mathrm{mag})\end{array}$ & $\begin{array}{c}R \\
(\mathrm{mag})\end{array}$ & $\begin{array}{c}S_{R} \\
(\mathrm{mag})\end{array}$ & $\begin{array}{c}I \\
(\mathrm{mag})\end{array}$ & $\begin{array}{c}S_{I} \\
(\mathrm{mag})\end{array}$ \\
\hline 2455065.01 & $17.304(039)$ & -0.110 & $16.865(033)$ & 0.010 & $16.957(034)$ & 0.020 & $16.889(092)$ & -0.020 \\
\hline 2455065.96 & $16.519(036)$ & -0.100 & $16.216(035)$ & 0.000 & $16.309(029)$ & 0.020 & $16.346(038)$ & -0.020 \\
\hline 2455068.02 & $15.115(036)$ & -0.050 & $15.151(028)$ & -0.010 & $15.117(035)$ & 0.010 & $15.273(037)$ & -0.010 \\
\hline 2455069.00 & $14.748(036)$ & -0.040 & $14.790(031)$ & -0.010 & $14.858(034)$ & 0.010 & $14.892(037)$ & -0.010 \\
\hline 2455070.00 & $14.614(037)$ & -0.040 & $14.546(029)$ & -0.010 & $14.592(029)$ & 0.010 & $14.652(037)$ & -0.010 \\
\hline 2455071.94 & $14.120(037)$ & -0.030 & $14.199(072)$ & -0.010 & $14.220(032)$ & 0.000 & $14.232(043)$ & -0.010 \\
\hline 2455072.96 & $13.972(037)$ & -0.030 & $14.042(028)$ & -0.010 & $14.137(029)$ & 0.010 & $14.146(037)$ & -0.010 \\
\hline 2455073.99 & $13.847(036)$ & -0.030 & $13.937(035)$ & -0.010 & $14.057(031)$ & 0.010 & $14.103(037)$ & -0.010 \\
\hline 2455074.94 & $13.825(036)$ & -0.030 & $13.851(028)$ & 0.000 & $13.986(029)$ & 0.010 & $14.059(037)$ & -0.010 \\
\hline 2455076.02 & $13.738(036)$ & -0.020 & $13.672(031)$ & 0.000 & $13.855(029)$ & 0.020 & $13.935(038)$ & -0.020 \\
\hline 2455077.00 & $13.609(036)$ & -0.030 & $13.623(028)$ & 0.000 & $\ldots$ & $\ldots$ & $13.870(038)$ & -0.020 \\
\hline 2455077.93 & $13.643(036)$ & -0.030 & $13.592(029)$ & 0.000 & $13.779(029)$ & 0.020 & $13.918(037)$ & -0.020 \\
\hline 2455079.89 & $13.629(036)$ & -0.040 & $13.537(028)$ & 0.000 & $13.692(029)$ & 0.030 & $13.880(037)$ & -0.020 \\
\hline 2455081.01 & $13.583(036)$ & -0.030 & $13.451(028)$ & 0.000 & $13.637(029)$ & 0.030 & $13.875(037)$ & -0.030 \\
\hline 2455081.92 & $13.622(036)$ & -0.030 & $13.494(030)$ & 0.000 & $13.695(029)$ & 0.030 & $13.940(037)$ & -0.030 \\
\hline 2455082.94 & $13.669(039)$ & -0.030 & $13.555(028)$ & 0.000 & $13.708(029)$ & 0.030 & $13.939(037)$ & -0.030 \\
\hline 2455083.94 & $13.595(036)$ & -0.030 & 13.487 (039) & 0.000 & $13.663(034)$ & 0.030 & $13.942(039)$ & -0.030 \\
\hline 2455084.99 & $13.765(036)$ & -0.030 & $13.586(029)$ & 0.000 & $13.749(029)$ & 0.040 & $14.032(040)$ & -0.030 \\
\hline 2455085.93 & $13.769(037)$ & -0.030 & $13.602(028)$ & 0.000 & $13.782(029)$ & 0.030 & $14.080(037)$ & -0.030 \\
\hline 2455087.94 & $13.861(037)$ & -0.030 & $13.629(028)$ & 0.000 & $13.941(029)$ & 0.030 & $14.179(038)$ & -0.040 \\
\hline 2455089.97 & $\ldots$ & $\ldots$ & $13.651(029)$ & -0.010 & $13.900(029)$ & 0.030 & $14.295(037)$ & -0.040 \\
\hline 2455090.92 & $13.989(036)$ & -0.030 & $13.768(028)$ & -0.010 & $14.006(029)$ & 0.020 & $14.337(038)$ & -0.040 \\
\hline 2455091.92 & $14.047(046)$ & -0.040 & $13.739(044)$ & -0.010 & $13.965(041)$ & 0.020 & $14.311(038)$ & -0.050 \\
\hline 2455092.96 & $14.123(038)$ & -0.040 & $13.815(034)$ & 0.000 & $14.125(029)$ & 0.020 & $14.438(037)$ & -0.050 \\
\hline 2455093.99 & $14.359(039)$ & -0.040 & $\ldots$ & $\ldots$ & $14.258(032)$ & 0.020 & $14.618(037)$ & -0.050 \\
\hline 2455094.87 & $14.416(037)$ & -0.021 & $14.055(028)$ & 0.000 & $14.334(029)$ & 0.010 & $14.625(037)$ & -0.050 \\
\hline 2455095.95 & $14.537(036)$ & -0.020 & $14.115(028)$ & 0.000 & $14.394(029)$ & 0.010 & $14.615(037)$ & -0.050 \\
\hline 2455097.02 & $14.639(038)$ & -0.020 & $14.085(030)$ & 0.000 & $14.345(029)$ & 0.010 & $14.606(037)$ & -0.050 \\
\hline 2455097.90 & $14.776(038)$ & -0.020 & $14.238(028)$ & 0.000 & $14.460(029)$ & 0.010 & $14.663(037)$ & -0.050 \\
\hline 2455098.93 & $14.885(036)$ & -0.030 & $14.203(028)$ & 0.010 & $14.482(029)$ & 0.010 & $14.621(037)$ & -0.060 \\
\hline 2455099.94 & $15.000(036)$ & -0.020 & $14.301(029)$ & 0.010 & $14.470(029)$ & 0.020 & $14.601(037)$ & -0.050 \\
\hline 2455100.93 & $15.113(042)$ & -0.030 & $\ldots$ & $\ldots$ & $14.444(031)$ & 0.020 & $14.515(037)$ & -0.050 \\
\hline 2455101.98 & $15.145(037)$ & -0.040 & $14.330(028)$ & 0.010 & $14.476(029)$ & 0.020 & $14.461(040)$ & -0.050 \\
\hline 2455102.96 & $15.175(036)$ & -0.050 & $14.335(028)$ & 0.010 & $14.425(029)$ & 0.020 & $14.435(037)$ & -0.040 \\
\hline 2455104.96 & $15.381(036)$ & -0.060 & $14.413(028)$ & 0.010 & $14.403(029)$ & 0.030 & $14.394(037)$ & -0.030 \\
\hline 2455106.87 & $15.591(039)$ & -0.050 & $14.524(029)$ & 0.010 & $14.425(029)$ & 0.030 & $14.434(037)$ & -0.020 \\
\hline 2455112.91 & $16.076(044)$ & -0.060 & $14.825(029)$ & 0.020 & $14.666(030)$ & 0.030 & $\ldots$ & $\ldots$ \\
\hline 2455114.90 & $16.112(037)$ & -0.060 & $14.928(038)$ & 0.020 & $14.781(029)$ & 0.020 & $14.430(037)$ & -0.010 \\
\hline 2455116.96 & $16.206(091)$ & -0.060 & $15.104(029)$ & 0.010 & $14.916(029)$ & 0.020 & $14.544(037)$ & -0.010 \\
\hline 2455120.93 & $16.288(036)$ & -0.080 & $15.292(028)$ & 0.010 & $15.066(031)$ & 0.020 & $14.754(037)$ & 0.000 \\
\hline 2455123.89 & $16.480(039)$ & -0.080 & $15.388(028)$ & 0.010 & $15.337(029)$ & 0.030 & $15.049(037)$ & -0.010 \\
\hline 2455126.91 & $16.418(036)$ & -0.060 & $15.448(043)$ & 0.010 & $15.363(030)$ & 0.030 & $15.169(037)$ & 0.000 \\
\hline 2455129.81 & $16.514(036)$ & -0.050 & $15.636(029)$ & 0.010 & $15.532(029)$ & 0.020 & $15.325(037)$ & 0.000 \\
\hline 2455132.86 & $16.619(047)$ & -0.050 & $15.721(031)$ & 0.010 & $15.572(029)$ & 0.020 & $15.442(038)$ & 0.010 \\
\hline 2455133.85 & $16.546(040)$ & -0.040 & $15.704(029)$ & 0.000 & $15.544(034)$ & 0.020 & $15.509(037)$ & 0.020 \\
\hline 2455139.91 & $16.716(063)$ & -0.040 & $15.941(044)$ & 0.000 & $15.809(088)$ & 0.020 & $15.880(061)$ & 0.020 \\
\hline 2455143.81 & $17.001(148)$ & -0.050 & $16.088(043)$ & 0.000 & $15.935(031)$ & 0.020 & $15.973(047)$ & 0.010 \\
\hline 2455148.89 & $16.792(044)$ & -0.020 & $16.081(038)$ & 0.010 & $16.074(029)$ & 0.010 & $16.063(037)$ & 0.010 \\
\hline 2455154.82 & $16.974(037)$ & 0.000 & $16.316(029)$ & 0.010 & $16.326(029)$ & 0.000 & $16.397(038)$ & 0.010 \\
\hline 2455159.77 & $16.983(036)$ & 0.020 & $16.376(029)$ & 0.000 & $16.402(029)$ & 0.000 & $16.490(040)$ & 0.010 \\
\hline 2455168.81 & $17.187(045)$ & 0.040 & $16.682(033)$ & 0.000 & $\ldots$ & $\ldots$ & $16.820(038)$ & 0.010 \\
\hline 2455173.83 & $17.295(037)$ & 0.030 & $16.745(030)$ & 0.000 & $16.835(030)$ & -0.010 & $16.981(040)$ & 0.010 \\
\hline 2455182.76 & $17.286(036)$ & 0.060 & $16.994(030)$ & 0.000 & $17.066(041)$ & -0.010 & $17.385(127)$ & 0.010 \\
\hline 2455188.71 & $17.527(036)$ & 0.050 & $17.095(033)$ & 0.000 & $17.263(031)$ & -0.010 & $17.509(069)$ & 0.010 \\
\hline 2455196.79 & $17.589(061)$ & 0.060 & $\ldots$ & $\ldots$ & $\ldots$ & $\ldots$ & $\ldots$ & $\ldots$ \\
\hline
\end{tabular}

Notes. Photometry given in magnitudes with $1 \sigma$ (photometric and calibration) uncertainties (in mmag) presented in parentheses. The noted $S$-corrections have been applied to the photometry.

\subsection{Optical Spectroscopy}

We obtained low- and medium-resolution optical spectra of SN 2009ig with the Kast double spectrograph (Miller \& Stone 1993) on the Shane $3 \mathrm{~m}$ telescope at Lick Observatory, the
Low Resolution Spectrograph (LRS; Hill et al. 1998) on the Hobby-Eberly Telescope (HET) in queue mode (Shetrone et al. 2007), the Blue Channel spectrograph (Schmidt et al. 1989) on the $6.5 \mathrm{~m}$ MMT telescope, the Low Resolution Imaging 
Table 3

Swift/UVOT Photometry of SN 2009ig

\begin{tabular}{|c|c|c|c|}
\hline JD & Filter & Magnitude & $\begin{array}{c}S \text {-Correction } \\
\text { (mag) }\end{array}$ \\
\hline 2455065.33 & {$[U V W 2]$} & $20.575(288)$ & $\ldots$ \\
\hline 2455067.04 & {$[U V W 2]$} & $19.632(121)$ & $\ldots$ \\
\hline 2455068.21 & [UVW2] & $18.753(100)$ & $\ldots$ \\
\hline 2455068.92 & {$[U V W 2]$} & $18.328(060)$ & $\ldots$ \\
\hline 2455070.92 & {$[U V W 2]$} & 17.437 (029) & $\ldots$ \\
\hline 2455073.00 & [UVW2] & $16.886(030)$ & $\ldots$ \\
\hline 2455075.13 & {$[U V W 2]$} & $16.590(027)$ & $\ldots$ \\
\hline 2455076.46 & {$[U V W 2]$} & $16.551(064)$ & $\ldots$ \\
\hline 2455076.58 & [UVW2] & $16.513(029)$ & $\ldots$ \\
\hline 2455078.17 & {$[U V W 2]$} & $16.519(017)$ & $\ldots$ \\
\hline 2455079.33 & {$[U V W 2]$} & $16.544(029)$ & $\ldots$ \\
\hline 2455081.04 & {$[U V W 2]$} & $16.608(022)$ & $\ldots$ \\
\hline 2455081.96 & {$[U V W 2]$} & $16.665(016)$ & $\ldots$ \\
\hline 2455082.54 & {$[U V W 2]$} & $16.621(089)$ & $\ldots$ \\
\hline 2455084.42 & {$[U V W 2]$} & $16.747(031)$ & $\ldots$ \\
\hline 2455086.75 & {$[U V W 2]$} & $16.954(032)$ & $\ldots$ \\
\hline 2455088.92 & {$[U V W 2]$} & $17.160(027)$ & $\ldots$ \\
\hline 2455089.50 & {$[U V W 2]$} & $17.149(039)$ & $\ldots$ \\
\hline 2455098.96 & {$[U V W 2]$} & $18.295(071)$ & $\ldots$ \\
\hline 2455105.96 & {$[U V W 2]$} & $18.761(089)$ & $\ldots$ \\
\hline 2455107.88 & {$[U V W 2]$} & $19.067(113)$ & $\ldots$ \\
\hline 2455113.79 & {$[U V W 2]$} & $19.199(112)$ & $\ldots$ \\
\hline 2455116.88 & {$[U V W 2]$} & $19.297(122)$ & $\ldots$ \\
\hline 2455065.33 & [UVM2] & $>19.156$ & $\ldots$ \\
\hline 2455067.00 & [UVM2] & $>20.587$ & $\ldots$ \\
\hline 2455068.25 & [UVM2] & $19.828(240)$ & $\ldots$ \\
\hline 2455068.83 & [UVM2] & 20.345 (307) & $\ldots$ \\
\hline 2455070.92 & [UVM2] & $18.968(072)$ & $\ldots$ \\
\hline 2455073.00 & [UVM2] & $18.212(090)$ & $\ldots$ \\
\hline 2455075.13 & [UVM2] & $17.750(073)$ & $\ldots$ \\
\hline 2455076.63 & [UVM2] & $17.558(073)$ & $\ldots$ \\
\hline 2455079.33 & [UVM2] & $17.392(066)$ & $\ldots$ \\
\hline 2455080.63 & [UVM2] & $17.337(057)$ & $\ldots$ \\
\hline 2455082.50 & [UVM2] & $17.422(141)$ & $\cdots$ \\
\hline 2455084.42 & [UVM2] & $17.369(074)$ & $\ldots$ \\
\hline 2455086.75 & [UVM2] & $17.714(070)$ & $\ldots$ \\
\hline 2455089.50 & [UVM2] & $17.849(080)$ & $\ldots$ \\
\hline 2455098.96 & [UVM2] & $18.912(147)$ & $\cdots$ \\
\hline 2455105.96 & [UVM2] & $19.480(203)$ & $\ldots$ \\
\hline 2455107.88 & [UVM2] & $19.578(220)$ & $\ldots$ \\
\hline 2455113.79 & [UVM2] & $20.346(326)$ & $\ldots$ \\
\hline 2455116.88 & [UVM2] & $>20.100$ & $\ldots$ \\
\hline 2455065.29 & {$[U V W 1]$} & $19.384(221)$ & $\ldots$ \\
\hline 2455067.00 & {$[U V W 1]$} & $18.245(092)$ & $\ldots$ \\
\hline 2455068.25 & {$[U V W 1]$} & $17.131(056)$ & $\ldots$ \\
\hline 2455068.83 & {$[U V W 1]$} & $16.636(038)$ & $\ldots$ \\
\hline 2455070.50 & {$[U V W 1]$} & $15.631(026)$ & $\ldots$ \\
\hline 2455072.96 & {$[U V W 1]$} & $15.007(017)$ & $\ldots$ \\
\hline 2455075.13 & {$[U V W 1]$} & $14.787(016)$ & $\ldots$ \\
\hline 2455076.13 & {$[U V W 1]$} & $14.795(010)$ & $\ldots$ \\
\hline 2455076.58 & {$[U V W 1]$} & $14.782(018)$ & $\ldots$ \\
\hline 2455079.33 & {$[U V W 1]$} & $14.856(018)$ & $\ldots$ \\
\hline 2455080.58 & {$[U V W 1]$} & $14.921(017)$ & $\ldots$ \\
\hline 2455084.42 & {$[U V W 1]$} & $15.213(021)$ & $\ldots$ \\
\hline 2455086.75 & {$[U V W 1]$} & $15.427(021)$ & $\ldots$ \\
\hline 2455088.46 & {$[U V W 1]$} & $15.641(054)$ & $\ldots$ \\
\hline 2455089.50 & {$[U V W 1]$} & $15.728(027)$ & $\cdots$ \\
\hline 2455098.96 & {$[U V W 1]$} & $16.846(049)$ & $\ldots$ \\
\hline 2455101.83 & {$[U V W 1]$} & $17.145(073)$ & $\ldots$ \\
\hline 2455105.92 & {$[U V W 1]$} & $17.609(072)$ & $\ldots$ \\
\hline 2455107.88 & {$[U V W 1]$} & $17.713(081)$ & $\ldots$ \\
\hline 2455113.79 & {$[U V W 1]$} & $18.035(090)$ & $\ldots$ \\
\hline 2455116.88 & {$[U V W 1]$} & $18.481(123)$ & $\ldots$ \\
\hline 2455065.33 & $U$ & $17.896(068)$ & $\ldots$ \\
\hline 2455066.58 & $U$ & $16.712(042)$ & $\ldots$ \\
\hline
\end{tabular}

Table 3

(Continued)

\begin{tabular}{|c|c|c|c|}
\hline JD & Filter & Magnitude & $\begin{array}{c}S \text {-Correction } \\
\text { (mag) }\end{array}$ \\
\hline 2455068.58 & $U$ & $15.163(021)$ & $\ldots$ \\
\hline 2455070.50 & $U$ & $14.257(015)$ & $\ldots$ \\
\hline 2455072.96 & $U$ & $13.741(011)$ & $\ldots$ \\
\hline 2455075.13 & $U$ & $13.504(011)$ & $\ldots$ \\
\hline 2455076.58 & $U$ & $13.498(012)$ & $\ldots$ \\
\hline 2455079.33 & $U$ & $13.517(012)$ & $\ldots$ \\
\hline 2455081.00 & $U$ & $13.702(006)$ & $\ldots$ \\
\hline 2455084.42 & $U$ & $13.827(012)$ & $\ldots$ \\
\hline 2455086.75 & $U$ & $13.994(012)$ & $\ldots$ \\
\hline 2455088.92 & $U$ & $14.264(005)$ & $\ldots$ \\
\hline 2455089.50 & $U$ & $14.289(015)$ & $\ldots$ \\
\hline 2455098.96 & $U$ & $15.385(024)$ & $\ldots$ \\
\hline 2455065.33 & $B$ & $17.079(040)$ & -0.047 \\
\hline 2455066.58 & $B$ & $16.113(032)$ & -0.031 \\
\hline 2455068.58 & $B$ & $14.961(025)$ & -0.022 \\
\hline 2455070.50 & $B$ & $14.403(024)$ & -0.011 \\
\hline 2455072.96 & $B$ & $14.048(022)$ & -0.012 \\
\hline 2455075.12 & $B$ & $13.776(022)$ & -0.008 \\
\hline 2455076.58 & $B$ & $13.714(023)$ & -0.002 \\
\hline 2455079.33 & $B$ & $13.613(022)$ & -0.003 \\
\hline 2455080.58 & $B$ & $13.666(022)$ & -0.005 \\
\hline 2455084.42 & $B$ & $13.720(022)$ & -0.005 \\
\hline 2455086.75 & $B$ & $13.842(022)$ & -0.008 \\
\hline 2455089.50 & $B$ & $14.002(023)$ & -0.010 \\
\hline 2455098.96 & $B$ & $14.845(025)$ & -0.011 \\
\hline 2455105.96 & $B$ & $15.483(028)$ & -0.024 \\
\hline 2455107.88 & $B$ & $15.626(030)$ & -0.027 \\
\hline 2455113.79 & $B$ & $16.015(030)$ & -0.039 \\
\hline 2455116.88 & $B$ & $16.188(033)$ & -0.025 \\
\hline 2455065.33 & $V$ & $16.546(052)$ & 0.031 \\
\hline 2455066.58 & $V$ & $15.827(042)$ & 0.027 \\
\hline 2455068.58 & $V$ & $14.926(031)$ & 0.017 \\
\hline 2455070.50 & $V$ & $14.411(028)$ & 0.026 \\
\hline 2455073.00 & $V$ & $14.010(024)$ & 0.017 \\
\hline 2455075.13 & $V$ & $13.816(024)$ & 0.032 \\
\hline 2455076.63 & $V$ & $13.697(025)$ & 0.038 \\
\hline 2455079.33 & $V$ & $13.561(024)$ & 0.036 \\
\hline 2455080.63 & $V$ & $13.568(024)$ & 0.034 \\
\hline 2455084.42 & $V$ & $13.602(024)$ & 0.033 \\
\hline 2455086.75 & $V$ & $13.654(024)$ & 0.044 \\
\hline 2455089.50 & $V$ & $13.748(025)$ & 0.047 \\
\hline 2455098.96 & $V$ & $14.273(028)$ & 0.043 \\
\hline 2455105.96 & $V$ & $14.515(029)$ & 0.037 \\
\hline 2455107.88 & $V$ & $14.631(030)$ & 0.037 \\
\hline 2455113.79 & $V$ & $14.925(031)$ & 0.041 \\
\hline 2455116.88 & $V$ & $15.199(034)$ & 0.036 \\
\hline
\end{tabular}

Notes. Photometry given in magnitudes with $1 \sigma$ uncertainties (in mmag) presented in parentheses. The noted $S$-corrections have been applied to the photometry.

Spectrometer (LRIS; Oke et al. 1995) on the $10 \mathrm{~m}$ Keck I telescope, and the DEep Imaging Multi-Object Spectrograph (DEIMOS; Faber et al. 2003) on the $10 \mathrm{~m}$ Keck II telescope. Spectra were typically made at low air mass and at the parallactic angle (Filippenko 1982). A log of our observations is given in Table 4.

Standard CCD processing and spectrum extraction were accomplished with IRAF. The data were extracted using the optimal algorithm of Horne (1986). Low-order polynomial fits to calibration-lamp spectra were used to establish the wavelength scale, and small adjustments derived from 
Table 4

Log of Optical Spectral Observations

\begin{tabular}{|c|c|c|c|c|}
\hline Phase $^{\mathrm{a}}$ & UT Date & Telescope/Instrument & $\begin{array}{c}\text { Exposure } \\
\text { (s) }\end{array}$ & Observer $^{\mathrm{b}}$ \\
\hline-14.2 & 2009 Aug 22.508 & Lick/Kast & 1800 & MC \\
\hline-14.1 & 2009 Aug 22.628 & Keck/LRIS & 300 & AS, HS \\
\hline-14.1 & 2009 Aug 22.635 & Keck/DEIMOS & $2 \times 120,2 \times 60$ & ET, RB, RG \\
\hline-13.3 & 2009 Aug 23.428 & HET/LRS & 900 & SO \\
\hline-13.1 & 2009 Aug 23.623 & Keck/DEIMOS & $4 \times 300$ & ET, RB, RG \\
\hline-12.2 & 2009 Aug 24.518 & Lick/Kast & 900 & VB \\
\hline-12.1 & 2009 Aug 24.638 & Keck/DEIMOS & $3 \times 300$ & $\mathrm{ET}, \mathrm{RB}, \mathrm{RG}$ \\
\hline-11.2 & 2009 Aug 25.522 & Lick/Kast & 900 & VB \\
\hline-11.1 & 2009 Aug 25.632 & Keck/DEIMOS & 300 & $\mathrm{ET}, \mathrm{JK}, \mathrm{RB}$ \\
\hline-10.3 & 2009 Aug 26.495 & MMT/Blue Channel & 180 & GW \\
\hline-10.1 & 2009 Aug 26.637 & Keck/DEIMOS & $3 \times 300$ & $\mathrm{ET}, \mathrm{JK}, \mathrm{RB}$ \\
\hline-9.3 & 2009 Aug 27.490 & MMT/Blue Channel & 180 & $\mathrm{FV}, \mathrm{LJ}$ \\
\hline-9.3 & 2009 Aug 27.499 & Lick/Kast & 600 & $\mathrm{SBC}, \mathrm{DP}$ \\
\hline-8.3 & 2009 Aug 28.493 & MMT/Blue Channel & 180 & $\mathrm{FV}, \mathrm{LJ}$ \\
\hline-8.2 & 2009 Aug 28.530 & Lick/Kast & 1500 & AM, JS, MK \\
\hline-7.4 & 2009 Aug 29.421 & HET/LRS & 450 & SR \\
\hline-7.3 & 2009 Aug 29.495 & MMT/Blue Channel & 120 & $\mathrm{FV}, \mathrm{LJ}$ \\
\hline-6.4 & 2009 Aug 30.407 & HET/LRS & 450 & SR \\
\hline-6.3 & 2009 Aug 30.440 & MMT/Blue Channel & 120 & $\mathrm{FV}, \mathrm{LJ}$ \\
\hline-5.4 & 2009 Aug 31.402 & HET/LRS & 550 & $\mathrm{JC}$ \\
\hline-5.3 & 2009 Aug 31.480 & MMT/Blue Channel & 120 & GW \\
\hline-3.4 & 2009 Sep 02.402 & HET/LRS & 450 & $\mathrm{JC}$ \\
\hline-2.3 & 2009 Sep 03.490 & HET/LRS & 450 & SO \\
\hline-1.4 & 2009 Sep 04.472 & $\mathrm{HET} / \mathrm{LRS}$ & 450 & $\mathrm{SO}$ \\
\hline-0.4 & 2009 Sep 05.404 & HET/LRS & 450 & SO \\
\hline
\end{tabular}

Notes.

a Days since $B$ maximum, 2009 September 6.0 (JD 2,455,080.5).

b $\mathrm{AM}=\mathrm{A}$. Morton, $\mathrm{AS}=\mathrm{A}$. Stockton, $\mathrm{SBC}=\mathrm{S} . \mathrm{B}$. Cenko, DP = D. Poznanski, ET = E. Tollerud, FV = F. Vilas, GW = G. Williams, HS = H.-Y. Shih, JC = J. Caldwell, JK = J. Kalirai, JS = J. Silverman, LJ = L. Jiang, $M C=M$. Childress, $M K=M$. Kandrashoff, $R B=R$. Beaton, $R G=R$. Guhathakurta, $S O=S$. Odewahn, $\mathrm{SR}=\mathrm{S}$. Rostopchin, $\mathrm{VB}=\mathrm{V}$. Bennert.

night-sky lines in the object frames were applied. The DEIMOS data were reduced using a modified version of the DEEP2 datareduction pipeline, ${ }^{18}$ which bias corrects, flattens, rectifies, and sky subtracts the data (Foley et al. 2007). We employed our own IDL routines to flux-calibrate the data and remove telluric lines using the well-exposed continua of the spectrophotometric standards (Wade \& Horne 1988; Matheson et al. 2000; Foley et al. 2003). Our optical spectra are presented in Figure 3.

\subsection{Ultraviolet Spectroscopy}

SN 2009ig was observed with the UV grism on Swift/UVOT for eight pointings totaling 82,608 s between 2009 August 23.7 and 2009 September 14.4. The UVOT grism has spectral resolution $R=150$ and a wavelength accuracy of about $7 \AA$. The observations were obtained in "clocked" mode, which limits contamination by unrelated zero orders at wavelengths longer than $\sim 3200 \AA$. The grism reduction software had several updates compared to that used by Bufano et al. (2009), including a wavelength equation that varied with detector position, an extraction slit that followed the small spectral curvature, and the use of a ninth-order polynomial to better follow the rapidly changing background. The gross spectrum was extracted using a slit of width 13 pixels, and the background was extracted within 11 pixels of the gross slit to minimize contamination by the galaxy nucleus. Due to an unfavorable roll angle, the background of the first two spectra was contaminated by a bright $\mathrm{F}$ star, but

\footnotetext{
18 http://astro.berkeley.edu/cooper/deep/spec $2 \mathrm{~d} /$.
}

Table 5

Log of Swift/UVOT Spectral Observations

\begin{tabular}{ccr}
\hline \hline Phase $^{\mathrm{a}}$ & UT Date & $\begin{array}{c}\text { Exposure } \\
(\mathrm{s})^{\mathrm{b}}\end{array}$ \\
\hline-13.0 & 2009 Aug 23.72 & 5742 \\
-12.0 & 2009 Aug 24.72 & 6601 \\
-11.1 & 2009 Aug 25.66 & 1491 \\
-9.2 & 2009 Aug 27.57 & 13803 \\
-4.2 & 2009 Sep 1.65 & 5764 \\
-2.1 & 2009 Sep 3.70 & 13318 \\
1.5 & 2009 Sep 7.34 & 17775 \\
8.5 & 2009 Sep 14.43 & 19259 \\
\hline \multicolumn{4}{c}{ Notes. } & \\
a Days since $B$ maximum, 2009 & September 6.0 \\
(JD 2,455,080.5). \\
b Exposure times are corrected for dead time.
\end{tabular}

there was no obvious contamination of any other spectra. To reduce the contamination from the $\mathrm{F}$ star, background regions starting only 1 pixel from the $\mathrm{SN}$ were chosen for the first two spectra. The contamination is minimal, but there is still some slight oversubtraction of flux for these spectra. Details of our observations are presented in Table 5.

\section{BASIC OBSERVATIONAL DATA}

SN 2009ig is a very well observed SN. Using our extensive photometry, we provide basic parameters for it. Fitting a 


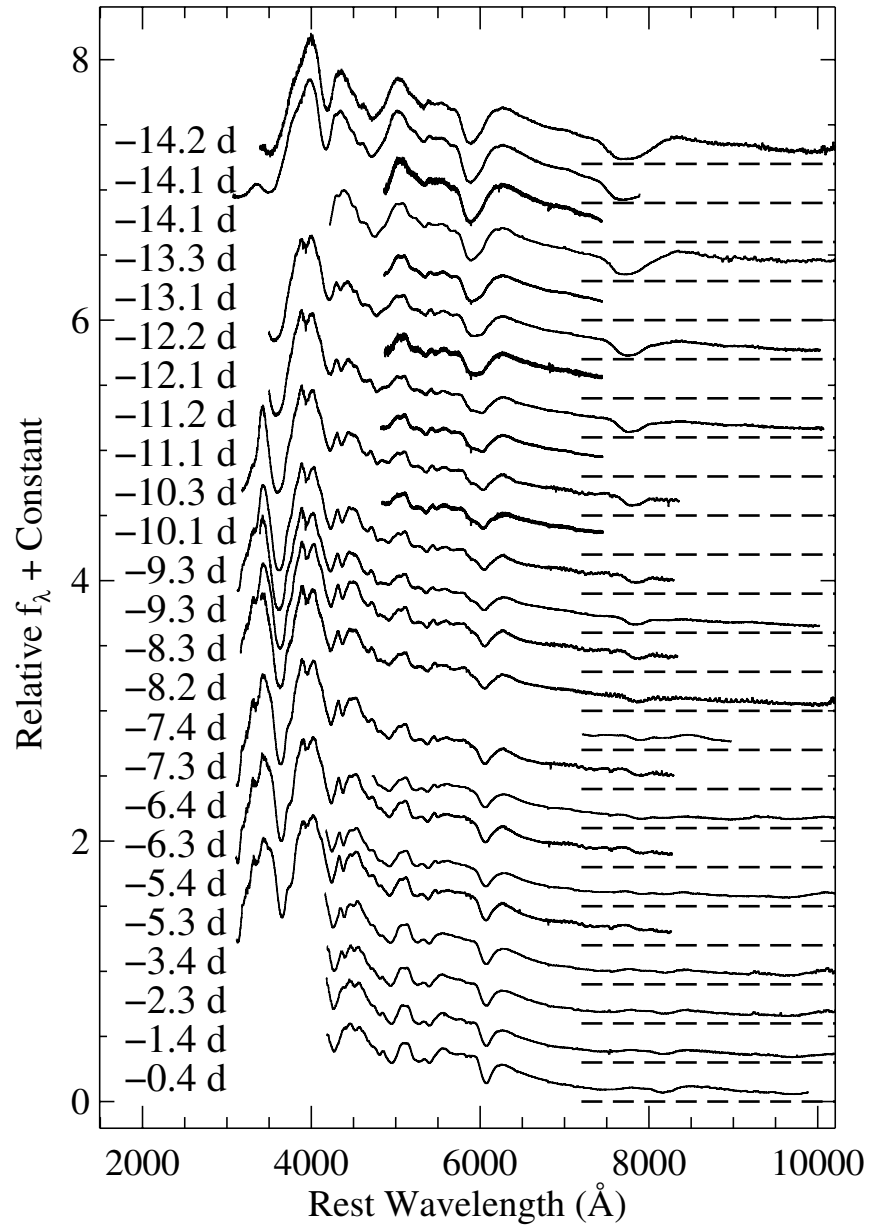

Figure 3. Optical spectra of SN 2009ig. The spectra are denoted by their phase relative to maximum brightness in the $B$ band. The zero-flux level is indicated for each spectrum by a dashed line.

polynomial through a restricted region of each light curve, we determine the maximum-light characteristics presented in Table 6.

SN 2009ig peaked at $V=13.52 \mathrm{mag}$, making it one of the brightest $\mathrm{SNe}$ Ia of the last decade. The decline rate, $\Delta m_{15}(B)=$ $0.89 \mathrm{mag}$, indicates that it was a slightly slower decliner than a nominal SN Ia with $\Delta m_{15}=1.1$ mag. Using Milky Way reddening values (Schlegel et al. 1998) and the assumed distance modulus, but neglecting any potential host-galaxy reddening, we find that SN 2009ig had a peak absolute magnitude of $M_{V}=-19.19 \pm 0.40 \mathrm{mag}$. Fitting the KAIT $B V R I$ light curves with MLCS2k2 (Jha et al. 2007), we find $\mu=32.96 \pm 0.02 \mathrm{mag}$ (assuming $H_{0}=74 \mathrm{~km} \mathrm{~s}^{-1} \mathrm{Mpc}^{-1}$ ), consistent with the Tully-Fisher measurement of $\mu=32.6 \pm 0.4$ mag (Tully 1988).

The MLCS2k2 fit also finds a host-galaxy extinction of $A_{V}=$ $0.01 \pm 0.01 \mathrm{mag}$, consistent with no host-galaxy reddening. Examination of the optical spectra shows that there is somewhat

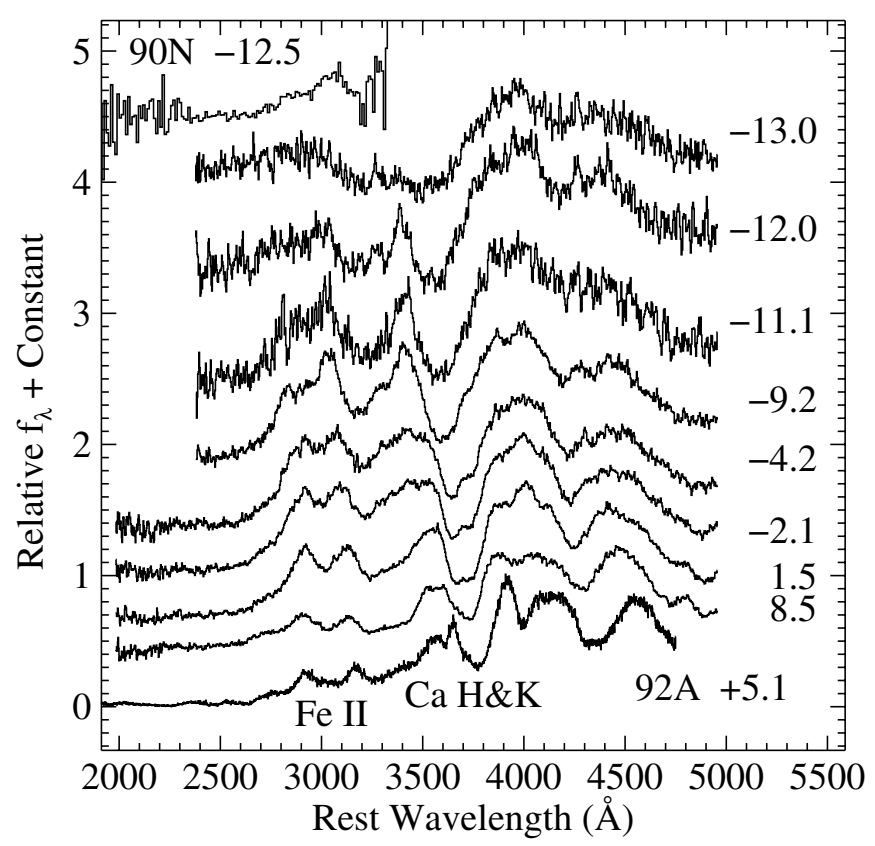

Figure 4. UV spectra of SN 2009ig. The spectra are denoted by their phase relative to maximum brightness in the $B$ band. Also plotted are the previously earliest spectrum of an SN Ia, SN $1990 \mathrm{~N}$ at $t=-12.5$ days (Leibundgut et al. 1991), and the earliest high-quality, true UV spectrum of an SN Ia, SN 1992A at $t=5.1$ days (Kirshner et al. 1993).

strong Na I D absorption (equivalent width $0.4 \AA$ ) at the redshift of the SN. However, this level of absorption is consistent with zero host-galaxy reddening as determined from large samples of SNe Ia (Blondin et al. 2009; Folatelli et al. 2010; Poznanski et al. 2011).

\section{ULTRAVIOLET SPECTROSCOPY}

Under our program to get Swift UV spectra of nearby SNe Ia (GI-5080130; PI Filippenko), we obtained eight low-resolution UV spectra of SN 2009ig. Six spectra were from before maximum brightness, a $38 \%$ increase in the published premaximum UV spectra of SNe Ia. The first spectrum was taken -13.0 days before $B$ maximum, making it the earliest UV spectrum ever obtained of an SN Ia. The SN was spectroscopically followed by Swift until 8.5 days after maximum brightness. We present our UV spectra in Figure 4. The earliest spectrum shows a very broad absorption feature spanning 3000-4000 ̊; however, it separates into two distinct features (Co II and $\mathrm{Ca} \mathrm{H} \& \mathrm{~K}$ ) only a day later. The UV continuum (2500-3000 $⿱$ ) $)$ is high in the first spectrum, declining over the next $2-4$ days, at which point it is relatively similar for all following epochs. The background region of the first spectrum was slightly contaminated by a nearby star (see Section 2.3), but this has the effect of reducing the continuum.

Table 6

Photometric Information for SN 2009ig

\begin{tabular}{|c|c|c|c|c|c|c|c|c|}
\hline Filter & {$[U V W 2]$} & [UVM2] & {$[U V W 1]$} & $U$ & $B$ & $V$ & $R$ & $I$ \\
\hline $\begin{array}{l}\text { JD of max. } \\
-2,455,000\end{array}$ & $78.30 \pm 0.05$ & $81.30 \pm 0.05$ & $76.76 \pm 0.05$ & $76.57 \pm 0.08$ & $80.54 \pm 0.04$ & $82.17 \pm 0.03$ & $81.33 \pm 0.29$ & $78.26 \pm 0.25$ \\
\hline Mag at max. & $16.50 \pm 0.04$ & $17.31 \pm 0.14$ & $14.75 \pm 0.04$ & $13.46 \pm 0.01$ & $13.66 \pm 0.03$ & $13.52 \pm 0.02$ & $13.64 \pm 0.02$ & $13.88 \pm 0.03$ \\
\hline Peak abs. mag & $-16.30 \pm 0.40$ & $-15.55 \pm 0.42$ & $-18.02 \pm 0.40$ & $-19.32 \pm 0.40$ & $-19.08 \pm 0.40$ & $-19.19 \pm 0.40$ & $-19.05 \pm 0.40$ & $-18.78 \pm 0.40$ \\
\hline$\Delta m_{15}(\mathrm{mag})$ & $1.03 \pm 0.03$ & $1.49 \pm 0.10$ & $1.10 \pm 0.03$ & $0.95 \pm 0.01$ & $0.89 \pm 0.02$ & $0.58 \pm 0.01$ & $0.69 \pm 0.01$ & $0.63 \pm 0.02$ \\
\hline
\end{tabular}






Figure 5. Blueshifted velocity of the minimum of the UV Fe II feature as a function of time for various $\mathrm{SNe}$ Ia assuming a rest-frame $g f$-weighted wavelength of $3250 \AA$. The normal, low-, and high-luminosity (as defined by Foley et al. 2008) SNe Ia are shown in black, red, and blue (with solid, dotted, and dashed lines connecting points), respectively.

(A color version of this figure is available in the online journal.)

The absorption feature at $\sim 3000 \AA$, attributed to Fe II $\lambda 3250$ (Branch \& Venkatakrishna 1986), is possibly present at $t \leqslant$ -9.2 days, although it is quite weak. Even at $t=-4.2$ days, it is not particularly strong. However, at $t=-2.1$ days, it has become rather strong, and by $t=1.5$ days, it is very strong, similar to that of other SNe Ia (Foley et al. 2008).

The Fe II $\lambda 3250$ feature is the strongest one in the UV. Its velocity and pseudo-equivalent width ( $\mathrm{pEW}$ ) evolution have been measured for several objects by Foley et al. (2008), who found that the velocity evolution is similar for all objects, but the strength of the feature near maximum brightness correlates with light-curve shape. We present the velocity and $\mathrm{pEW}$ measurements for SN 2009ig relative to the Foley et al. (2008) sample in Figures 5 and 6, respectively. SN 2009ig appears to follow the previously established trends; its velocity is consistent with that of other SNe Ia, while its $\mathrm{pEW}$ is small, as expected given its relatively broad light curve.

Overall, the UV spectral evolution of SN 2009ig is similar to that of other normal SNe Ia. Near maximum brightness, we measure a UV ratio $\left(\mathcal{R}_{\mathrm{UV}}=f_{\lambda}(2770 \AA) / f_{\lambda}(2900 \AA)\right.$; Foley et al. 2008) of 0.23 , consistent with SN 2009ig having a relatively broad light curve. The shape of the SN Ia UV SED is determined by several factors related to the progenitor and explosion. High-quality UV spectroscopy can disentangle these effects (Sauer et al. 2008). Unfortunately, the relatively low-S/N data obtained at $\lambda<2500 \AA$ preclude detailed modeling of the UV spectrum similar to what was done by Sauer et al. (2008).

\section{EARLY-TIME PHOTOMETRY}

\subsection{Rise Time}

The first detection of SN 2009ig was 16.4 days before $B$ maximum, and therefore the rise time for SN 2009ig was $\geqslant 16.4$ days. The last nondetection was 20.2 days before $B$ maximum. However, extrapolating the light curve in any reasonable manner, the last nondetection is not sufficiently deep to be particularly constraining for the rise time. Nonetheless, SN 2009ig is one of the earliest ever detected SNe Ia.

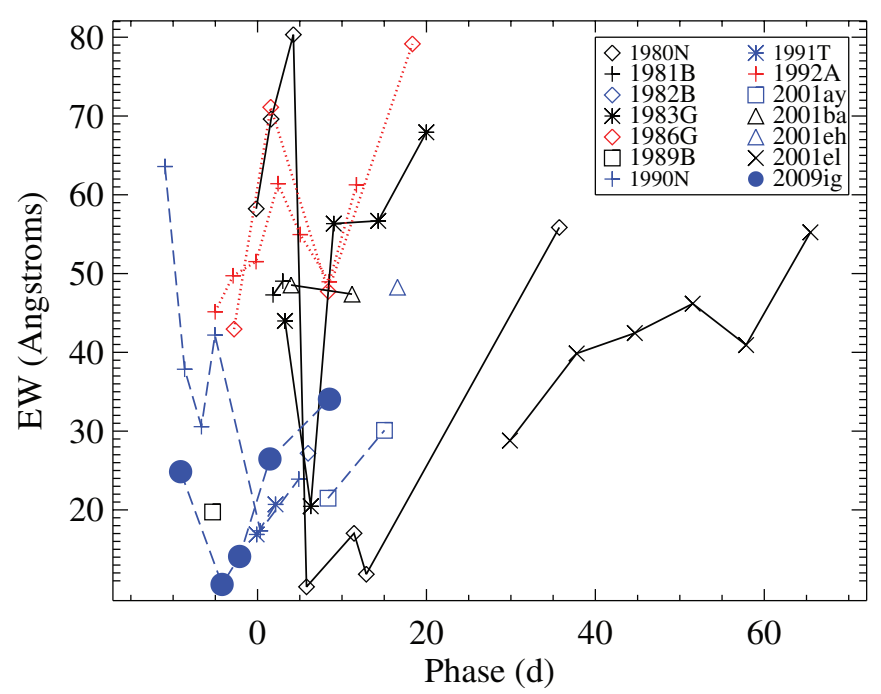

Figure 6. $\mathrm{pEW}$ of the Fe II feature as a function of time for various $\mathrm{SNe}$ Ia. The normal, low-, and high-luminosity (as defined by Foley et al. 2008) SNe Ia are shown in black, red, and blue (with solid, dotted, and dashed lines connecting points), respectively.

(A color version of this figure is available in the online journal.)

Figure 7 shows the light curves of SN 2009ig, shifted such that the data represent the brightness below peak. Assuming that an $\mathrm{SN}$ is approximately a homologously expanding blackbody at early times, the luminosity of the SN should increase as a function of $\tau^{2}$, where $\tau$ is the time after explosion. This form assumes that there is negligible temperature evolution at early times, although Arnett (1982) showed that this form should be independent of temperature at sufficiently early times. Given the strong color evolution at early times (Section 5.2), this model may not apply to SN 2009ig.

Using a similar method to that of Riess et al. (1999), but without stretching the light curve, we fit the $B$-band data with $t<$ -10 days to determine the rise time, $t_{r}$. The data are best fit with $t_{r}=17.14 \pm 0.04$ days, where the uncertainty is only statistical. This fit is shown in Figure 7. However, there is a potential systematic difference in the measurement if the SN is not well described by a homologously expanding blackbody. Differences from this assumption should be larger at later times. If we examine only the first three KAIT data points (corresponding to $15.4,14.5$, and 12.4 days before $B$ maximum), the best-fit rise time is $t_{r}=17.11 \pm 0.07$ days, which is consistent with the other value. Nonetheless, we take the conservative approach of averaging the two values and using the larger uncertainty for our final measurement: $t_{r}=17.13 \pm 0.07$ days. Our first detection at $t=-16.4$ days means that SN 2009ig was discovered $\sim 17 \mathrm{hr}$ after explosion. Measured rise times for SN 2009ig in all optical bands are consistently $\sim 17$ days. All photometric data indicate that SN 2009ig was discovered $\lesssim 1$ day after explosion.

Ganeshalingam et al. (2011) examined a large sample of lowredshift SN Ia light curves, focusing on their rise time. This sample included the $B$ and $V$ KAIT light curves of SN 2009ig that are presented here. However, the SN 2009ig light curves were not well fit by their template light curve, and SN 2009ig was rejected from their final analysis.

The rise time for SN 2009ig is relatively normal for an SN Ia. Hayden et al. (2010b) determined that the average Sloan Digital Sky Survey (SDSS) SN Ia rise time is $17.38 \pm 0.17$ days (with a standard deviation in the sample of 1.8 days) in the $B$ band. Strovink (2007) found an average rise time of $17.44 \pm 0.39$ days 


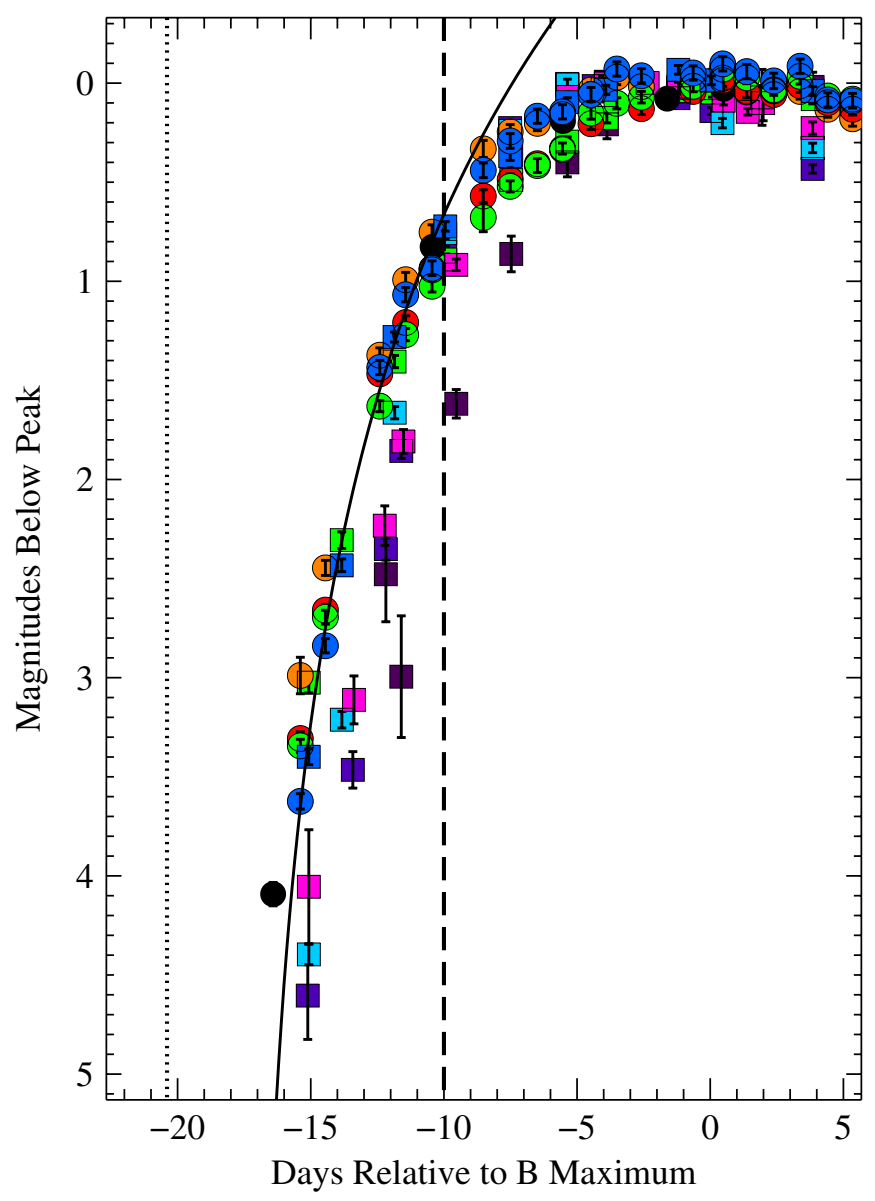

Figure 7. [UVW2][UVM2][UVW1] $U B V R I$ (fuchsia, dark purple, navy, cyan, blue, green, red, and orange, respectively; circles and squares correspond to KAIT and Swift/UVOT data, respectively) and unfiltered (black circles, with the label "Unf") light curves of SN 2009ig. The data have been shifted such that maximum brightness in each band corresponds to zero magnitude. The dotted line shows the time of the last nondetection, while the dashed line corresponds to $t=-10$ days. The solid curve represents the best-fit rise-time function for the $B$ band with $t<-10$ days; the best-fit rise time is $t_{r}=17.13$ days.

(A color version of this figure is available in the online journal.)

for a small sample of low-redshift SNe Ia with excellent premaximum light curves. SN 2009ig has high-velocity ejecta and a high velocity gradient for Si II $\lambda 6355$ (see Section 6.1), and Pignata et al. (2008) suggested that high velocity gradient $\mathrm{SNe}$ Ia have shorter rise times than low velocity gradient SNe. Ganeshalingam et al. (2011) found that low-redshift high-velocity SNe Ia had a rise time of $16.63 \pm 0.29$ days. Observations of SN 2009ig support the trend that high-velocity $\mathrm{SNe}$ Ia tend to have shorter rise times in the $B$ band.

Hayden et al. (2010b) and Ganeshalingam et al. (2011) note that their rise-time values are shorter than those found in previous studies (e.g., Riess et al. $1999, t_{r}=19.98 \pm 0.15$ days), but the differences are related to the adopted template light curve and a single-stretch (rather than a two-stretch) method. Our method does not stretch the SN 2009ig light curves; we measure the true rise time of SN 2009ig.

SN 2009ig declined in the $B$ band by 1.1 mag in 17.05 days; therefore, the difference between the rise and fall time for SN 2009ig is $t_{r}-t_{f}=0.08$ days. This value is consistent with the majority of objects in the Hayden et al. (2010b) sample, but is slightly shorter than average. On the other hand, Ganeshalingam et al. (2011) found an average value of

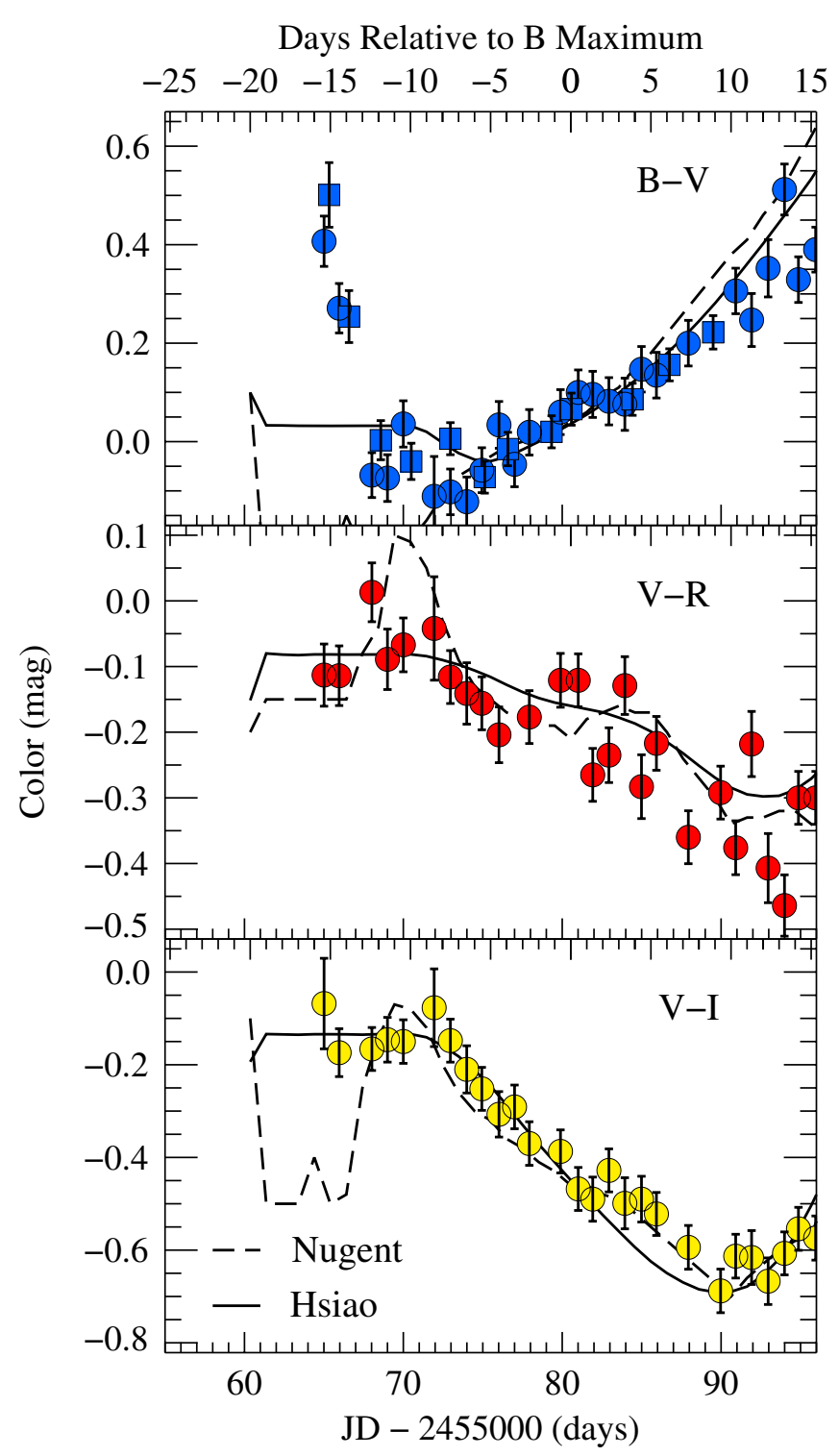

Figure 8. $B-V, V-R$, and $V-I$ color curves of SN 2009ig. All photometry has been corrected for the Milky Way reddening of $E(B-V)=0.032 \mathrm{mag}$ (Schlegel et al. 1998). The solid and dashed lines represent the color curves of the Hsiao et al. (2007) and Nugent et al. (2002) template light curves, respectively, where each has been shifted to match SN 2009ig at maximum brightness.

(A color version of this figure is available in the online journal.)

$t_{r}-t_{f}=1.55 \pm 0.27$ days for high-velocity SNe Ia. The scatter is larger than the quoted uncertainty in the mean, but there are few $\mathrm{SNe}$ in their sample that have $t_{r}-t_{f}<1$ day.

\subsection{Color Curves}

Examining the optical color curves shown in Figure 8, we see significant color evolution in $B-V$ for $t<-10$ days (this was also noted by Ganeshalingam et al. 2011). From $t=-15.2$ days to $t=-11.2$ days, $B-V$ decreased from $0.58 \mathrm{mag}$ to $0.04 \mathrm{mag}$, a decline of 0.14 mag day ${ }^{-1}$. The color changes in $V-R$ and $V-I$ are relatively small at early times $(\lesssim 0.2 \mathrm{mag})$.

The UV color curves (Figure 9) also show very fast color evolution at early times. [UVW2] $-V$, [UVM2] $-V$, and $[U V W 1]-V$ all become redder by $\sim 1.5 \operatorname{mag}$ from $t \approx-15$ days to $t \approx-5$ days. This is similar to the $U-V$ behavior, which is not unexpected given the effective wavelengths of the filters for an SN Ia SED. The color evolution is similar to the trends seen 


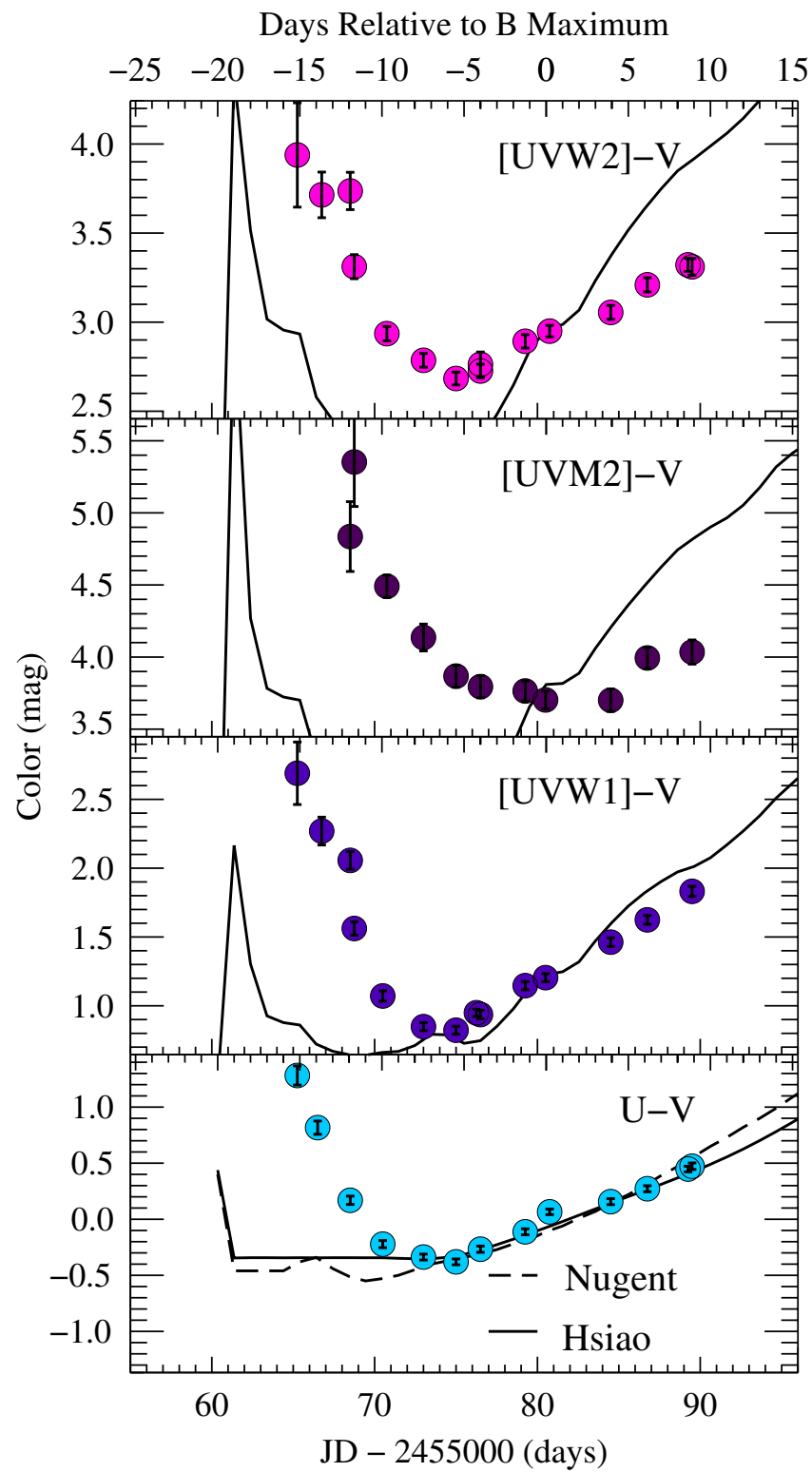

Figure 9. [UVW2] $V,[U V M 2]-V,[U V W 1]-V$, and $U-V$ color curves of SN 2009ig (all obtained with Swift). All photometry has been corrected for the Milky Way reddening of $E(B-V)=0.032 \mathrm{mag}$ (Schlegel et al. 1998) and the reddening corrections of Brown et al. (2010) for the Swift bands. The solid and dashed lines represent the color curves of the Hsiao et al. (2007) and Nugent et al. (2002) template light curves, respectively, where each has been shifted to match SN 2009ig at maximum brightness.

(A color version of this figure is available in the online journal.)

with a larger sample (Milne et al. 2010), but SN 2009ig extends these trends to much earlier phases.

Figures 8 and 9 compare the color curves of SN 2009ig to those of the commonly used SN Ia template light curves of Nugent et al. (2002) and Hsiao et al. (2007). After shifting to match the colors of SN 2009ig at maximum brightness, the templates reproduce the optical colors of SN 2009ig quite well for $t>-5$ days. The Nugent et al. (2002) and Hsiao et al. (2007) templates deviate from SN 2009ig for $t \lesssim-6$ and -9 days, respectively, in $B-V$. The $V-R$ colors appear to be more consistent out to the earliest observations of SN 2009ig, but there may be a significant deviation at $t \approx-10$ days. The Nugent et al. (2002) template matches the $V-I$ color of SN 2009ig at even its earliest epochs, but the Hsiao et al. (2007) template deviates significantly at $t \lesssim-12$ days.

There are no Nugent et al. (2002) template light curves for the UV bands, but we are able to synthesize light curves in the Swift bands from the Hsiao et al. (2007) template spectra using the in-orbit filter functions (Poole et al. 2008). The template matches SN 2009ig in $U-V$ and [UVWI] $-V$ after $t \approx-5$ days but is significantly bluer at earlier times, similar to $B-V$. The template light curves are generally very poor in $[U V W 2]-V$ and [UVM2] $-V$ at all times, but are also bluer than the SN 2009ig data before maximum brightness (when normalizing to the color at maximum brightness). The long red tails for the UV filters should not directly affect the synthesized light curves if the filter definitions are correct. However, the tails can significantly affect the color curves depending on the reddening.

Since the Hsiao et al. (2007; Nugent et al. 2002) (and to a lesser extent at this point, the Nugent et al. 2002) template is used for comparing data to a standard template and generating $K$-corrections for high-redshift SN Ia light curves, its fidelity is important. Clearly, improvements can be made at early times. Nonetheless, the exact colors of the template are not important for $K$-corrections (since the template SED is warped to match the observed colors), but we show in Section 6.1 that there are significant differences between SN 2009ig and the Hsiao et al. (2007) template spectrum in the spectral features at early times. Interestingly, Hayden et al. (2010b) present a $K$-corrected composite SDSS SN Ia $B-V$ color curve (their Figure 11) that has $B-V \approx 0 \pm 0.08$ mag for $t \leqslant-10$ days, which is significantly different from what is seen in SN 2009ig, but is consistent with the Hsiao et al. (2007) colors. Although SN 2009ig may be a significant outlier, it is likely that the $B-V$ color measured by Hayden et al. (2010b) was significantly affected by $K$-corrections which used the Hsiao et al. (2007) template. Similar to the above cases, light-curve fitters, which also use template light curves and SEDs, should consider these potential problems.

\subsection{Emission from Interaction with a Companion Star}

There is a simple observational prediction for an SN Ia that comes from a single-degenerate progenitor system where the companion star fills its Roche lobe: emission from the interaction of the SN ejecta with the companion star that can dominate over the light produced from radioactive decay at early times (Kasen 2010). The exact characteristics of the emission, particularly the peak luminosity and duration, depend on the progenitor system and viewing angle. Relative to the SN light curve, this emission should be brightest in the UV. The effect strongly depends on viewing angle, where significant excess emission is observed when the viewing angle is aligned such that the companion star is between the WD and the observer, and essentially no additional emission is generated for viewing angles $180^{\circ}$ from the WD-companion-observer configuration. For all companions explored by Kasen (2010) (a $1 \mathrm{M}_{\odot}$ red giant, and 2 and $6 \mathrm{M}_{\odot}$ main-sequence stars-all undergoing Roche-lobe overflow so that the radius of the companion is approximately half the separation distance), we would expect some excess emission in the $B$ band at $\tau \approx 2$ days if we observed the SN from $\sim 10^{\circ}$ from the direction of the companion; the UV bands should display an even stronger signal.

In Figures 2 and 7, we see no indication of early excess emission in the optical bands. However, there is some indication of excess emission at early times in the UV. Because of their relatively low $\mathrm{S} / \mathrm{N}$, the [UVM2] data are not particularly 
constraining. However, the [UVW2] and [UVW1] data suggest some excess emission. We caution that because of the long red tail in these bands, the apparent excess emission may be the result of optical color evolution.

A strong indication that there is no detected interaction is that the $B-V$ and UV colors of SN 2009ig at early times (our earliest measurement is $\sim 0.7$ day after explosion) are relatively red and get bluer until $t \approx-10$ days. This color evolution is not expected for the Kasen (2010) models.

To place further limits on excess emission at early times, the Kasen (2010) models are examined in more detail. The models produced are for the radioactively powered and interaction powered components combined. Since the Kasen (2010) models do not precisely reproduce the radioactively powered light curves, we have decided to examine the difference between the light curves from various viewing angles and the model light curve from a viewing angle farthest from the companion $\left(167^{\circ}\right)$. There may be excess emission from the interaction with the companion even in this model, so the difference between the models can be considered a lower limit on the expected emission for a given viewing angle.

Figure 10 shows the SN 2009ig light-curve data after subtracting an expanding fireball model. As explained above, this should be a reasonable model for the early-time behavior of an $\mathrm{SN}$ Ia. For this comparison, we fit the data with $t<-10$ days relative to $B$ maximum, but ignored the first data point in each band. The strongest signal should be in the earliest data. This results in a measurement of the observed flux above that expected from modeling the additional data. If there is detectable excess flux in other observations, then this should be apparent as systematic residuals to the expanding fireball model.

Because of their numerical limitations, the Kasen (2010) models have significant scatter in their flux from one epoch to another, corresponding to $\sim 0.2 \mathrm{mag}$ for each band. This scatter must be included in any potential detection. We define our threshold as three times the geometric mean of the model scatter and the photometric uncertainty of the earliest data point in each band. This detection limit is marked as a dashed line in Figure 10.

The first $V$-band observation is consistent with the expanding fireball model. The first $B$-band observation is slightly brighter than expected from the expanding fireball model, but is not above our threshold. The first $U$-band measurement is significantly above our threshold, but only two data points were used to constrain the model. Both the [UVW1] and [UVW2] bands show significant excess in their first observations relative to the expanding fireball model. However, the models were fit with only three data points in each of these bands, so the fits may be biased. Nonetheless, this trend of excess flux in the UV bands is similar to what was seen in Figures 2 and 7. Furthermore, all bands bluer than $V$ have excess flux in their first data point and the data slightly after $t=-10$ days are consistent with the model, making an excess more likely.

Using the Kasen (2010) model SEDs, we were able to construct residual light curves in each of our bands. Using the $B$ threshold, we can place a limit on the possible viewing angle for the three progenitors considered. The $1 M_{\odot}$ red giant, $6 M_{\odot}$ main-sequence, and $2 M_{\odot}$ main-sequence models with viewing angles (relative to the companion star) greater than $151^{\circ}, 109^{\circ}$, and $68^{\circ}$, respectively, are consistent with this measurement (i.e., they have excess flux below the threshold at the time of the earliest $B$-band observation). Given the distribution of possible viewing angles, these values corresponds to $<6 \%$,

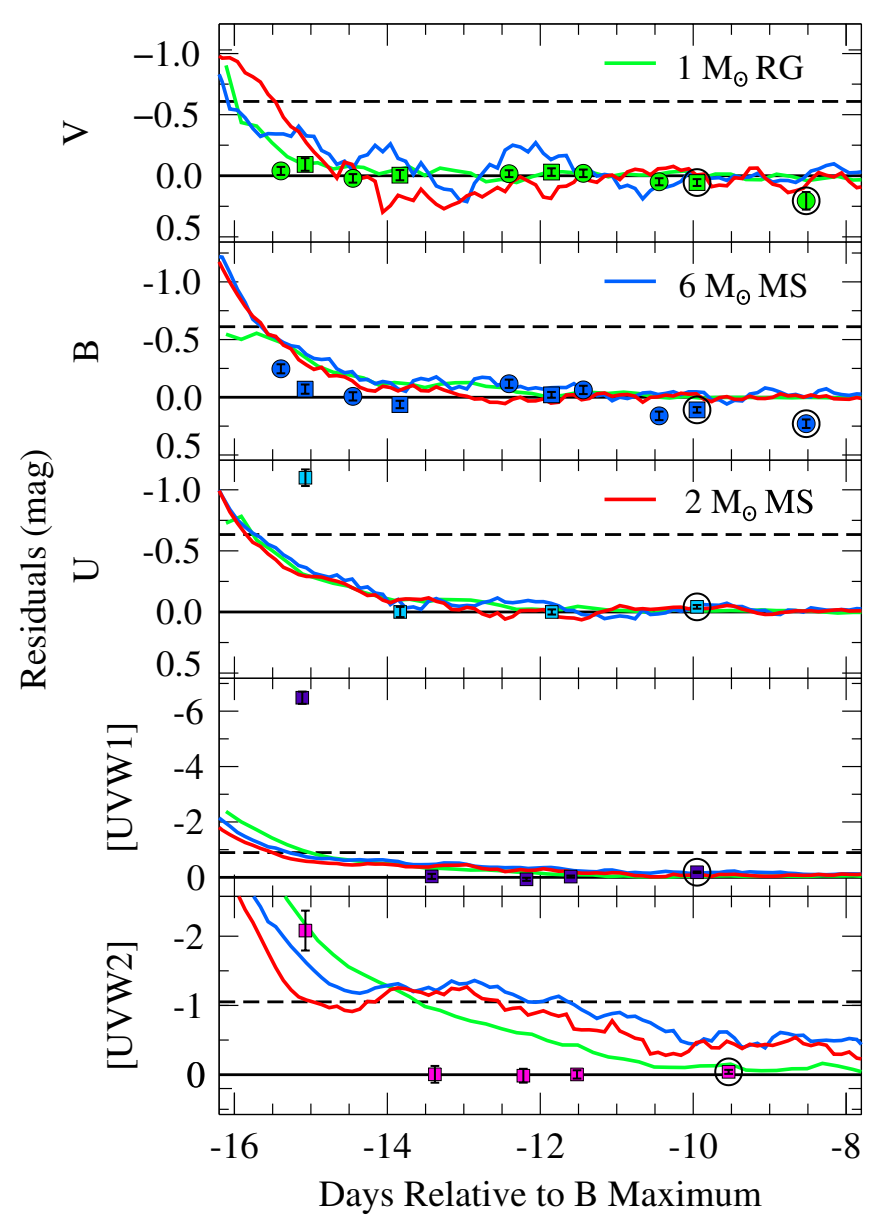

Figure 10. SN 2009ig [ $U V W 2][U V W 1] U B V$ photometry (bottom to top panels) with an expanding fireball model subtracted. The expanding fireball model was fit to the data at $-15<t<-10$ days relative to $B$ maximum, which excludes the first one or two data points. The circled points are at $t>-10$ days and are not included in the fit. The green, blue, and red curves correspond to excess emission in the $1 M_{\odot}$ red giant, $6 M_{\odot}$ main-sequence, and $2 M_{\odot}$ main-sequence companion Kasen (2010) models at viewing angles of $151^{\circ}, 109^{\circ}$, and $68^{\circ}$, respectively. Specifically, these curves are an indication of the emission from the interaction only. These lines correspond to the largest viewing angle (relative to the companion star) consistent with $B$-band observations of SN 2009ig (i.e., are below the threshold at the time of the earliest observation). The dashed black line represents the threshold above which a band has excess emission.

(A color version of this figure is available in the online journal.)

$34 \%$, and $69 \%$ of the random viewing angles. Despite being formally inconsistent with a simple expanding fireball model, the [UVW2] band is consistent with the Kasen (2010) models for the large angle considered above. The excess emission in the $U$ and [ $U V W 1]$ bands is also in excess of these models/angles. In fact, the [ $U V W 1]$ data are inconsistent with all models/angles investigated by Kasen (2010).

The excess flux seen in the bluer bands appears to be real. Supporting this view are that the difference from the expanding fireball model is always an excess, data just after the phase range we use to fit the model are consistent with the model, there appears to be a visual excess in the bluest bands, and including the first data point in a fireball fit significantly increases the $\chi^{2}$ per degree of freedom. However, the disagreement with the models in the various bands suggests that the simple Kasen (2010) model is not responsible for this excess. Instead, considering that the colors become bluer with time (the opposite of predictions) and the long tail of the UV filters, we suggest that the excess seen in the UV light curves is the result of 


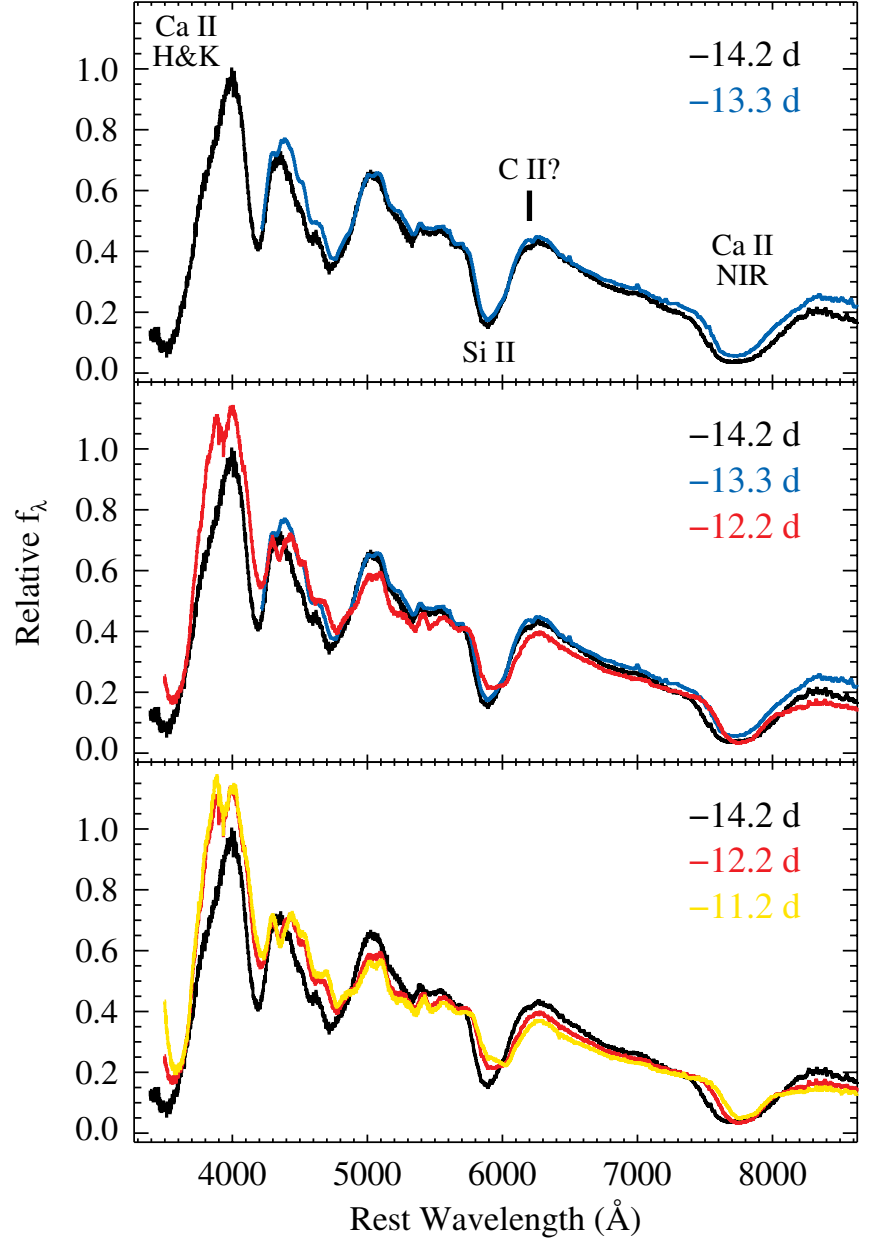

Figure 11. $t=-14.2$ (black), -13.3 (blue), -12.2 (red), and -11.2 day (yellow) spectra of SN 2009ig. Features discussed in the text are labeled. (A color version of this figure is available in the online journal.)

optical (and perhaps UV) color evolution. If there is significant color evolution because of either changes to spectral features or the temperature, then the expanding fireball model may not be sufficient for modeling the early-time light curve.

Using samples of $\sim 100$ SNe Ia from SDSS and Supernova Legacy Survey, respectively, Hayden et al. (2010a) and Bianco et al. (2011) produced $K$-corrected composite rest-frame $B$-band light curves. The ensemble of light curves did not show any deviation from a standard rise, indicating that red giant progenitor systems must be a small fraction (if there is any contribution at all) of the SN Ia progenitor systems. Ganeshalingam et al. (2011) examined 61 well-sampled low-redshift SN Ia light curves, finding no signs of interaction under simple assumptions; however, relaxing these assumptions, they found large systematic trends in the data, precluding any definitive conclusions. Unlike previous work that focused on the statistical treatment of large samples, we have concentrated on a single well-observed SN, which can reduce some potential systematic biases. Nevertheless, the SN 2009ig data provide additional concern when making simple assumptions about the early-time light curves of SNe Ia.

\section{OPTICAL SPECTROSCOPY}

\subsection{Early-time Spectroscopy}

In Figure 11, we show a detailed comparison of a subset of our earliest spectra. In the first panel, we compare spectra obtained
-14.2 and -13.3 days relative to $B$ maximum. Although these spectra are separated by only 1 day, there are some differences, particularly in the shape of the Ca II near-infrared (NIR) triplet. The second panel adds the spectrum at -12.2 days relative to $B$ maximum. This spectrum is significantly different from that taken only two days earlier. In particular, the Ca II NIR triplet and the Si II $\lambda 6355$ features have very different shapes. The Si II $\lambda 6355$ feature has a flatter bottom and is much weaker at $t=-12.2$ days. The velocity of these features (as well as others, including $\mathrm{Ca} \mathrm{H} \& \mathrm{~K}$ ) is significantly lower in the later spectrum. The continuum is also different, with the later spectrum being bluer, similar to what is seen in the $B-V$ color (Figure 8 ). The red wing of $\mathrm{Ca} \mathrm{H} \& \mathrm{~K}$ in the $t=-14.2$ day spectrum is non-Gaussian, with a change in the slope at $\sim 3800 \AA$. The Si II $\lambda 4130$ feature is not distinct in the earliest spectrum. By $t=-12.2$ days, this feature is clear. We speculate that at earlier times, this feature is at higher velocity and simply blends with $\mathrm{Ca} H \& \mathrm{~K}$, causing the peculiar red wing of $\mathrm{Ca} \mathrm{H} \& \mathrm{~K}$; however, it is possible that ionization effects suppress this feature at early times. The last panel of Figure 11 adds the $t=-11.2$ day spectrum. This spectrum is very similar to the $t=-12.2$ day spectrum in feature strength and continuum shape, but there is a noticeable difference in the velocities of the various features.

Parrent et al. (2011) analyzed two of the spectra presented here and attributed a small depression at $\sim 6200 \AA$ to $\mathrm{C}_{\text {II }} \lambda 6580$. Although we do not provide a detailed analysis of this feature, at $\sim 6200 \AA$, we note that it is present in all of our first six spectra corresponding to $t \leqslant-12.2$ days. Those spectra were obtained with four separate telescope/instrument configurations, so we believe that it is a real feature. It is also present in our $t=$ -11.1 day spectrum, but we do not have definitive detections for our $t=-12.1$ or -11.2 day spectra, though this is possibly the result of those spectra having a relatively low $\mathrm{S} / \mathrm{N}$. We do not detect this feature in any later epochs, giving additional credibility to it being real. If this feature is $\mathrm{C}_{\text {II }} \lambda 6580$, it would correspond to a velocity of about $-17,000 \mathrm{~km} \mathrm{~s}^{-1}$, making it much lower velocity than the velocity inferred from the minimum of Si II at the same epoch (about $-23,000 \mathrm{~km} \mathrm{~s}^{-1}$ ), but may be consistent with an extrapolation of the lowervelocity Si II component extrapolated to the earliest epochs (see Section 6.2). If $\mathrm{C}_{\mathrm{II}}$ is detected, this would be the first detection of it in a high-velocity $\mathrm{SN}$ Ia.

The spectra of SN 2009ig change dramatically until about 12 days before maximum brightness. After this time, the general spectral features and shape stay relatively constant until after maximum brightness. This epoch also approximately corresponds to the time when the $\mathrm{SN} B-V$ color evolution slows down (Section 5.2).

In Section 5.2, we showed that the early-time color evolution of SN 2009ig was poorly matched by the color evolution of the Hsiao et al. (2007) template spectra. However, that comparison is not apt for $K$-corrections and some other applications since it neglected to warp the template spectra to match the observed colors of SN 2009ig. Without warping, the Hsiao et al. (2007) template spectrum has different broadband colors than SN 2009ig. We fit a spline to the ratio of the broadband integrated fluxes of the template and SN spectra. The template spectrum is divided by the spline to warp the template spectrum to have the same UBVRI magnitudes of the combined UV/optical SN 2009ig spectrum. In the top panel of Figure 12, we show the $t=-13.0$ day Swift UV spectrum, the $t=-14.2$ day optical spectrum, and the $t=-14$ day $U B V R I$ color-warped Hsiao et al. (2007) template spectrum. There are 


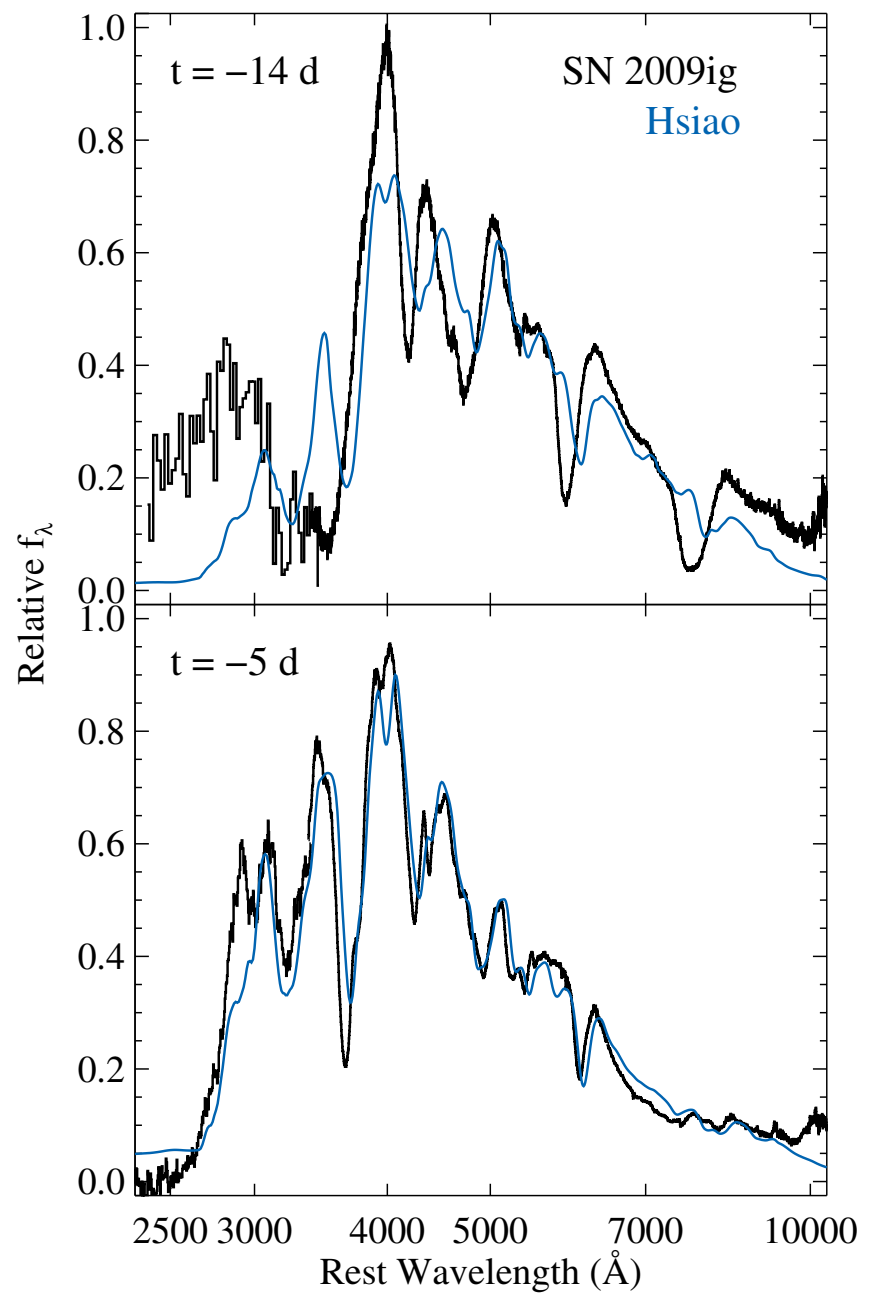

Figure 12. $t=-13.0$ day Swift UV and $t=-14.2$ day optical spectra (top panel) and the $t=-4.2$ days Swift UV and $t=-5.8$ days optical spectra (bottom panel) of SN 2009ig (black curves). The blue curves are the $t=-14$ day and $t=-5$ day $U B V R I$ color-warped Hsiao et al. (2007) template spectra (top and bottom panels, respectively).

(A color version of this figure is available in the online journal.)

several differences between the SN 2009ig and Hsiao et al. (2007) spectra. In particular, the Hsiao et al. (2007) spectrum has weaker, lower-velocity features and significant deviations for $\lambda<4000 \AA$.

The bottom panel of Figure 12 displays the $t=-4.2$ day Swift UV spectrum, the $t=-5.8$ day optical spectra, and the $t=-5$ day UBVRI color-warped Hsiao et al. (2007) template spectrum. At this phase, the Hsiao et al. (2007) template spectrum is a much better match to SN 2009ig; there are still some differences in the UV, particularly the feature at $2900 \AA$ and the continuum for $\lambda<2600 \AA$, but the match is significantly better than for the $t=-14$ day spectrum. Clearly, additional early-time data are necessary to improve the fidelity of template spectra and light curves. Use of the current template spectra will provide significantly discrepant $K$-corrections, even after five-band color warping.

\subsection{Si II $\lambda 6355$}

While the overall shape of the spectrum of SN 2009ig shows most of its evolution during the first few days after explosion, individual spectral features continue to evolve for several more days. An example of this is the strong Si II $\lambda 6355$ line. Using the

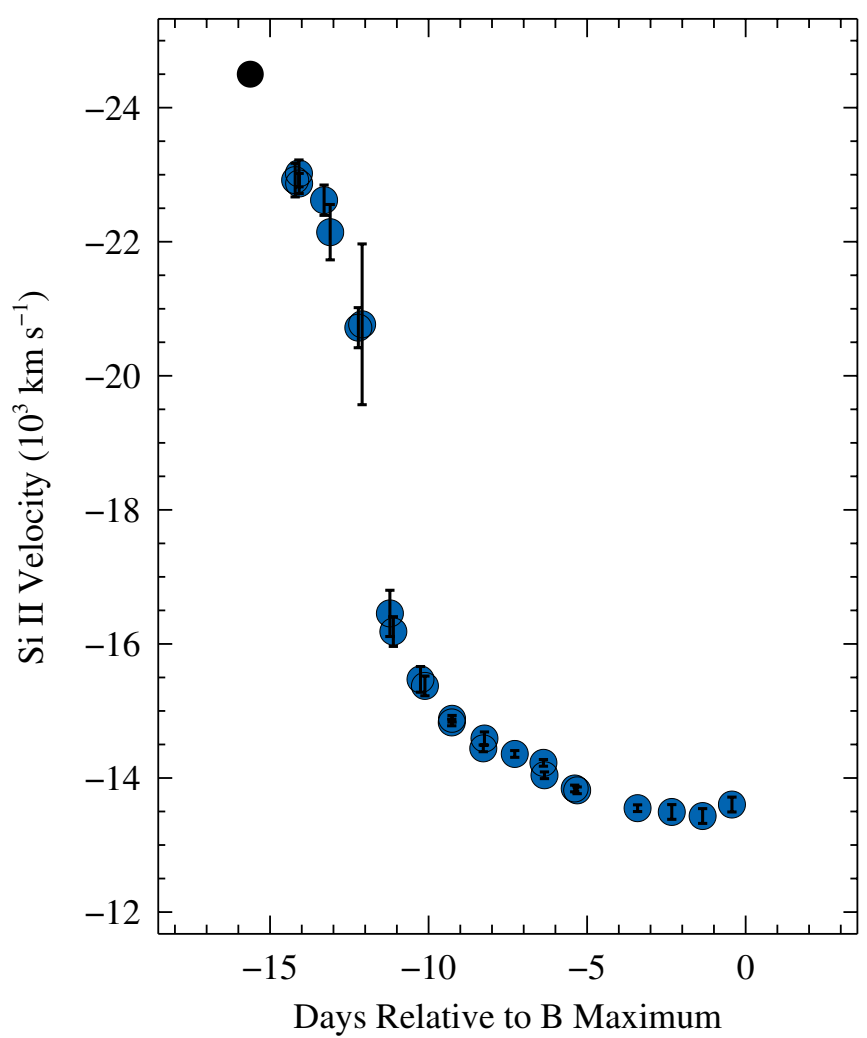

Figure 13. Velocity evolution of the Si II $\lambda 6355$ feature for SN 2009ig. The blue points come from our spectra. The black point was reported by Navasardyan et al. (2009).

(A color version of this figure is available in the online journal.)

method outlined by Foley et al. (2011, and references therein), we determined the wavelength of maximum absorption for this feature; we present a characteristic velocity of the line in Figure 13.

SN 2009ig displays the highest-velocity Si II $\lambda 6355$ ever published, with the earliest spectra having $v=-23,000 \mathrm{~km} \mathrm{~s}^{-1}$ at $t=-14.2$ days, significantly faster than the fastest measurements of SN 2006X $\left(-20,700 \mathrm{~km} \mathrm{~s}^{-1}\right.$ on $t=-11.6$ days; Quimby et al. 2006) and SN 2003W (-21,000 $\mathrm{km} \mathrm{s}^{-1}$ on $t=-11.2$ days; Foley et al. 2011). Navasardyan et al. (2009) reported an even higher velocity of $-24,500 \mathrm{~km} \mathrm{~s}^{-1}$ for SN 2009ig on $t=-15.6$ days. For our first spectrum, the blue wing of Si II $\lambda 6355$ extends to at least $5720 \AA$, corresponding to $v=-31,400 \mathrm{~km} \mathrm{~s}^{-1}$ or $>0.1 c$.

The velocity of the Si II $\lambda 6355$ feature evolves very quickly, decreasing by $5700 \mathrm{~km} \mathrm{~s}^{-1}$ in only 1.9 days. After this dramatic change, the velocity evolution appears to be linear in time, similar to that of most SNe Ia (Foley et al. 2011; J. M. Silverman et al. (2012, in preparation)). The velocity near maximum brightness is about $-13,500 \mathrm{~km} \mathrm{~s}^{-1}$, which is faster than $\sim 85 \%$ of all SNe Ia with $1 \leqslant \Delta m_{15}(B) \leqslant 1.5 \mathrm{mag}$ and faster than $\sim 85 \%$ of all SNe Ia with $\Delta m_{15}(B) \leqslant 1$ mag (Foley et al. 2011).

Further examination of the Si II $\lambda 6355$ feature demonstrates that the velocity evolution at early times is the result of multiple components. This is best displayed in Figure 14, which shows the Si II $\lambda 6355$ feature from spectra that occur before, during, and after this velocity change. At $t=-13.1$ days, there is some indication of two components, with the blue component dominating the profile. The relative strength quickly shifts; the components have similar strength at $t=-12.2$ days, but the 


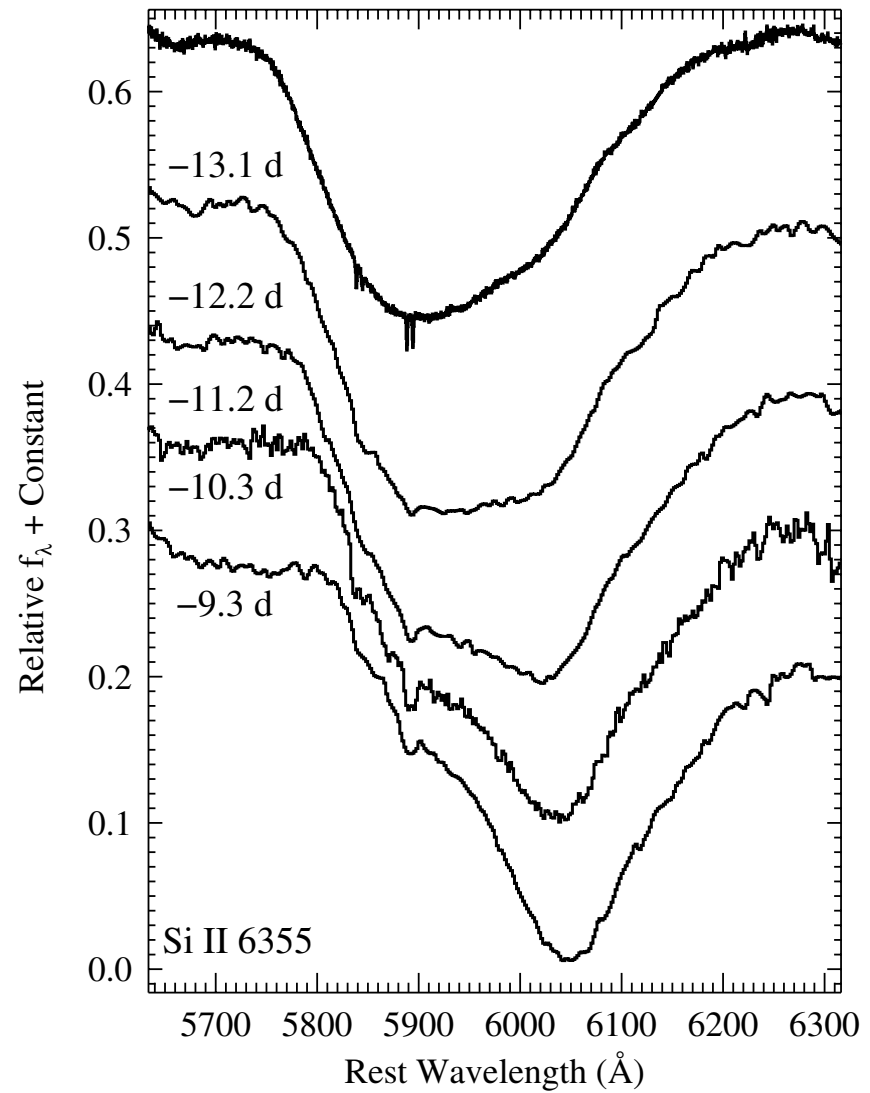

Figure 14. Spectra of SN 2009ig near the Si II $\lambda 6355$ feature. Phases are denoted for each spectrum.

red component is dominant by $t=-11.2$ days. This evolution continues to the point where the blue component is barely visible at $t=-9.3$ days. Further examination and discussion of the velocity evolution and line shapes of various features in the SN 2009ig spectra will be given by G. H. Marion et al. (2012, in preparation).

\section{DISCUSSION AND CONCLUSIONS}

SN 2009ig was discovered $\sim 17 \mathrm{hr}$ after explosion, relatively bright at peak ( $V=13.5 \mathrm{mag})$, and well positioned for detailed monitoring. We obtained densely sampled UV/optical light curves, an unparalleled UV spectral sequence, and an excellent, almost nightly optical spectral sequence from discovery through maximum brightness. Here we have focused on the early-time properties of this $\mathrm{SN}$, as well as on its unique UV spectral sequence.

SN 2009ig is a relatively normal SN Ia, although it is a slightly slow decliner $\left(\Delta m_{15}(B)=0.89 \mathrm{mag}\right)$ and has relatively fast ejecta at maximum brightness $\left(v_{\mathrm{Si}_{\text {II }}}^{0} \approx-13,500 \mathrm{~km} \mathrm{~s}^{-1}\right)$. We find that the rise time in the $B$ band is 17.13 days, similar to that of other high-velocity SNe Ia. The UV spectral features are similar to those of other SNe Ia. Our observations, particularly the UV spectra and the early-time photometry and spectroscopy, greatly expand the existing data at these wavelengths and phases.

Despite being a relatively normal SN Ia, early-time observations deviate from template light curves and spectra: SN 2009ig is redder than the templates. Even after color warping an earlytime template spectrum to match a spectrum of SN 2009ig at a similar phase, SN 2009ig has significantly broader, deeper, and higher-velocity spectral features than the template. The template also does a poor job at matching both the spectral features and the continuum at $\lambda<4000 \AA$. Distance estimates that rely on $K$-corrections and/or early-time data from templates could be biased, directly impacting cosmological measurements. Studies that rely on early-time $K$-corrected data, such as measuring the rise time (Aldering et al. 2000; Conley et al. 2006; Hayden et al. 2010b) and interaction with a binary companion (Hayden et al. 2010a; Bianco et al. 2011) of high-redshift SNe Ia, may be biased by these differences. Low-redshift studies should be minimally affected. An examination of the early-time light curve of SN 2009ig shows excess emission relative to a simple expanding fireball model $\left(L \propto \tau^{2}\right)$ in the UV bands. Although this behavior is expected if there is interaction between the $\mathrm{SN}$ and a binary companion, other predictions of the model (in particular, colors becoming redder) are not seen. It is therefore unlikely that we detect any interaction in the case of SN 2009ig.

SN 2009ig displays two velocity components in the Si II $\lambda 6355$ feature, with the higher-velocity component indicating the highest-velocity SN Ia ejecta ever observed. The relative strength of the components changes dramatically from $t=$ -13 days to $t=-9$ days, and one would not infer the presence of a high-velocity feature from spectroscopy after $t=-9$ days. Since there are few available spectra of SNe Ia at such early epochs, high-velocity Si II $\lambda 6355$ may be ubiquitous or at least very common, like high-velocity Ca II features (Mazzali et al. 2005).

In the age of giant synoptic surveys including that of the Large Synoptic Survey Telescope, SNe similar to SN 2009ig will still be rare. Detailed studies of SNe that are as nearby, bright, well positioned, and found as soon after explosion as SN 2009ig have the potential to provide as much or more information for constraining progenitor and explosion models than large samples of more distant SNe.

R.J.F. is supported by a Clay Fellowship. We thank the anonymous referee for informed comments and suggestions. We thank D. Kasen for useful discussions. We are grateful to the staffs at the Lick, Keck, HET, and MMT Observatories for their dedicated services. J. Bullock, J. Caldwell, M. Kandrashoff, A. Morton, P. Nugent, S. Odewahn, D. Poznanski, S. Rostopchin, H.-Y. Shih, F. Vilas, and G. Williams helped obtain some of the data presented here; we also thank J. Lee and D. Tytler for attempting to obtain data. Swift spectroscopic observations were performed under program GI-5080130; we are very grateful to N. Gehrels and the Swift team for executing the program quickly. Supernova research at Harvard is supported by NSF grant AST-0907903. A.V.F.'s supernova group at U.C. Berkeley is supported by NASA/Swift grants NNX09AG54G and NNX10AF52G, NSF grant AST-0908886, Gary and Cynthia Bengier, the Richard and Rhoda Goldman Fund, and the TABASGO Foundation. J.V. received support from Hungarian OTKA Grant K76816, NSF Grant AST-0707769, and Texas Advanced Research Project grant ARP-0094.

Some of the data presented herein were obtained at the W. M. Keck Observatory, which is operated as a scientific partnership among the California Institute of Technology, the University of California, and NASA; the observatory was made possible by the generous financial support of the W. M. Keck Foundation. The Hobby-Eberly Telescope (HET) is a joint project of the University of Texas at Austin, the Pennsylvania State University, Stanford University, Ludwig-MaximiliansUniversität München, and Georg-August-Universität Göttingen. The HET is named in honor of its principal benefactors, William 
P. Hobby and Robert E. Eberly. We acknowledge the use of public data from the Swift data archive. KAIT was constructed and supported by donations from Sun Microsystems, Inc., the Hewlett-Packard Company, AutoScope Corporation, Lick Observatory, the NSF, the University of California, the Sylvia \& Jim Katzman Foundation, and the TABASGO Foundation. This research has made use of the NASA/IPAC Extragalactic Database (NED), which is operated by the Jet Propulsion Laboratory, California Institute of Technology, under contract with NASA.

Facilities: HET(LRS), KAIT, Shane(Kast), Keck:I(LRIS), Keck:II(DEIMOS), MMT(Blue Channel), Swift(UVOT)

\section{REFERENCES}

Alard, C. 2000, A\&AS, 144, 363

Aldering, G., Knop, R., \& Nugent, P. 2000, AJ, 119, 2110

Amanullah, R., Lidman, C., Rubin, D., et al. 2010, ApJ, 716, 712

Arnett, W. D. 1982, ApJ, 253, 785

Bianco, F. B., Howell, D. A., Sullivan, M., et al. 2011, ApJ, 741, 20

Blondin, S., Prieto, J. L., Patat, F., et al. 2009, ApJ, 693, 207

Branch, D., \& Venkatakrishna, K. L. 1986, ApJ, 306, L21

Brown, P. J., Roming, P. W. A., Milne, P., et al. 2010, ApJ, 721, 1608

Bufano, F., Immler, S., Turatto, M., et al. 2009, ApJ, 700, 1456

Burrows, D. N., Hill, J. E., Nousek, J. A., et al. 2005, Space Sci. Rev., 120, 165

Colgate, S. A., \& McKee, C. 1969, ApJ, 157, 623

Conley, A., Guy, J., Sullivan, M., et al. 2011, ApJS, 192, 1

Conley, A., Howell, D. A., Howes, A., et al. 2006, AJ, 132, 1707

Cooke, J., Ellis, R. S., Sullivan, M., et al. 2011, ApJ, 727, L35

Faber, S. M., Phillips, A. C., Kibrick, R. I., et al. 2003, Proc. SPIE, 4841, 1657 Filippenko, A. V. 1982, PASP, 94, 715

Filippenko, A. V., Li, W. D., Treffers, R. R., \& Modjaz, M. 2001, in ASP Conf.

Ser. 246, IAU Colloq. 183, Small Telescope Astronomy on Global Scales, ed. B. Paczynski, W.-P. Chen, \& C. Lemme (San Francisco, CA: ASP), 121

Folatelli, G., Phillips, M. M., Burns, C. R., et al. 2010, AJ, 139, 120

Foley, R. J., Chornock, R., Filippenko, A. V., et al. 2009, AJ, 138, 376

Foley, R. J., Filippenko, A. V., \& Jha, S. W. 2008, ApJ, 686, 117

Foley, R. J., \& Kasen, D. 2011, ApJ, 729, 55

Foley, R. J., Narayan, G., Challis, P. J., et al. 2010, ApJ, 708, 1748

Foley, R. J., Papenkova, M. S., Swift, B. J., et al. 2003, PASP, 115, 1220

Foley, R. J., Sanders, N. E., \& Kirshner, R. P. 2011, ApJ, 742, 89

Foley, R. J., Smith, N., Ganeshalingam, M., et al. 2007, ApJ, 657, L105

Ganeshalingam, M., Li, W., Filippenko, A. V., et al. 2010, ApJS, 190, 418

Ganeshalingam, M., Li, W., \& Filippenko, A. V. 2011, MNRAS, 416, 2607

Gehrels, N., Chincarini, G., Giommi, P., et al. 2004, ApJ, 611, 1005

Hamuy, M., Phillips, M. M., Suntzeff, N. B., et al. 2003, Nature, 424, 651

Hayden, B. T., Garnavich, P. M., Kasen, D., et al. 2010a, ApJ, 722, 1691

Hayden, B. T., Garnavich, P. M., Kessler, R., et al. 2010b, ApJ, 712, 350

Hicken, M., Wood-Vasey, W. M., Blondin, S., et al. 2009, ApJ, 700, 1097

Hill, G. J., Nicklas, H. E., MacQueen, P. J., et al. 1998, Proc. SPIE, 3355, 375

Hillebrandt, W., \& Niemeyer, J. C. 2000, ARA\&A, 38, 191

Höflich, P., Wheeler, J. C., \& Thielemann, F.-K. 1998, ApJ, 495, 617

Horne, K. 1986, PASP, 98, 609
Howell, D. A. 2011, Nature Commun., 2, 350

Howell, D. A., Sullivan, M., Nugent, P. E., et al. 2006, Nature, 443, 308 Hoyle, F., \& Fowler, W. A. 1960, ApJ, 132, 565

Hsiao, E. Y., Conley, A., Howell, D. A., et al. 2007, ApJ, 663, 1187

Jeffery, D. J., Leibundgut, B., Kirshner, R. P., et al. 1992, ApJ, 397, 304

Jha, S., Kirshner, R. P., Challis, P., et al. 2006, AJ, 131, 527

Jha, S., Riess, A. G., \& Kirshner, R. P. 2007, ApJ, 659, 122

Kasen, D. 2010, ApJ, 708, 1025

Kessler, R., Becker, A. C., Cinabro, D., et al. 2009, ApJS, 185, 32

Kirshner, R. P., Jeffery, D. J., Leibundgut, B., et al. 1993, ApJ, 415, 589

Kleiser, I., Cenko, S. B., Li, W., \& Filippenko, A. V. 2009, CBET, 1918, 1

Landolt, A. U. 1992, AJ, 104, 340

Leibundgut, B., Kirshner, R. P., Filippenko, A. V., et al. 1991, ApJ, 371, L23

Lentz, E. J., Baron, E., Branch, D., \& Hauschildt, P. H. 2001, ApJ, 557, 266

Li, W., Filippenko, A. V., Chornock, R., et al. 2003, PASP, 115, 453

Li, W., Filippenko, A. V., Gates, E., et al. 2001, PASP, 113, 1178

Li, W., Jha, S., Filippenko, A. V., et al. 2006, PASP, 118, 37

Mandel, K. S., Narayan, G., \& Kirshner, R. P. 2011, ApJ, 731, 120

Matheson, T., Filippenko, A. V., Barth, A. J., et al. 2000, AJ, 120, 1487

Mazzali, P. A., Benetti, S., Altavilla, G., et al. 2005, ApJ, 623, L37

Miller, J. S., \& Stone, R. P. S. 1993, Lick Obs. Tech. Rep. 66 (Santa Cruz, CA: Lick Obs.)

Milne, P. A., Brown, P. J., Roming, P. W. A., et al. 2010, ApJ, 721, 1627

Navasardyan, H., Cappellaro, E., \& Benetti, S. 2009, CBET, 1918, 2

Nomoto, K., Thielemann, F.-K., \& Yokoi, K. 1984, ApJ, 286, 644

Nugent, P., Kim, A., \& Perlmutter, S. 2002, PASP, 114, 803

Oke, J. B., Cohen, J. G., Carr, M., et al. 1995, PASP, 107, 375

Parrent, J. T., Thomas, R. C., Fesen, R. A., et al. 2011, ApJ, 732, 30

Perlmutter, S., Aldering, G., Goldhaber, G., et al. 1999, ApJ, 517, 565

Pignata, G., Benetti, S., Mazzali, P. A., et al. 2008, MNRAS, 388, 971

Poole, T. S., Breeveld, A. A., Page, M. J., et al. 2008, MNRAS, 383, 627

Poznanski, D., Ganeshalingam, M., Silverman, J. M., \& Filippenko, A. V. 2011, MNRAS, 415, L81

Quimby, R., Brown, P., Gerardy, C., Odewahn, S. C., \& Rostopchin, S. 2006, CBET, 393, 1

Riess, A. G., Filippenko, A. V., Challis, P., et al. 1998, AJ, 116, 1009

Riess, A. G., Filippenko, A. V., Li, W., \& Schmidt, B. P. 1999, AJ, 118, 2668

Riess, A. G., Strolger, L.-G., Casertano, S., et al. 2007, ApJ, 659, 98

Roming, P. W. A., et al. 2005, Space Sci. Rev., 120, 95

Sauer, D. N., Mazzali, P. A., Blondin, S., et al. 2008, MNRAS, 391, 1605

Schlegel, D. J., Finkbeiner, D. P., \& Davis, M. 1998, ApJ, 500, 525

Schmidt, G. D., Weymann, R. J., \& Foltz, C. B. 1989, PASP, 101, 713

Shetrone, M., Cornell, M. E., Fowler, J. R., et al. 2007, PASP, 119, 556

Stritzinger, M., Hamuy, M., Suntzeff, N. B., et al. 2002, AJ, 124, 2100

Stritzinger, M., Suntzeff, N. B., Hamuy, M., et al. 2005, PASP, 117, 810

Strovink, M. 2007, ApJ, 671, 1084

Suzuki, N., Rubin, D., Lidman, C., et al. 2011, arXiv:1105.3470

Tully, R. B. 1988, in Nearby Galaxies Catalog (Cambridge: Cambridge Univ. Press), 221

Wade, R. A., \& Horne, K. 1988, ApJ, 324, 411

Wang, X., Filippenko, A. V., Ganeshalingam, M., et al. 2009a, ApJ, 699, L139

Wang, X., Li, W., Filippenko, A. V., et al. 2009b, ApJ, 697, 380

Wong, O. I., Ryan-Weber, E. V., Garcia-Appadoo, D. A., et al. 2006, MNRAS, 371,1855

Wood-Vasey, W. M., Miknaitis, G., Stubbs, C. W., et al. 2007, ApJ, 666, 694

Woosley, S. E., Taam, R. E., \& Weaver, T. A. 1986, ApJ, 301, 601 